\title{
U.S. Hydropower Resource Assessment for Illinois
}

\author{
Prepared by: \\ Alison M. Conner \\ James E. Francfort \\ Project Manager: \\ Ben N. Rinehart
}

Published January 1997

\section{Idaho National Engineering Laboratory Renewable Energy Products Department Lockheed Martin Idaho Technologies Company Idaho Falls, Idaho 83415}

Prepared for the

U.S. Department of Energy

Assistant Secretary for Energy Efficiency and Renewable Energy Under DOE Idaho Operations Office

Contract DE-AC07-94ID13223 


\section{DISCLAIMIER}

Portions of this doenment may be illegible in electronic image products. Images are produced from the best available original docmenert. 


\begin{abstract}
The U.S. Department of Energy is developing an estimate of the undeveloped hydropower potential in the United States. The Hydropower Evaluation Software (HES) is a computer model that was developed by the Idaho National Engineering Laboratory for this purpose. HES measures the undeveloped hydropower resources available in the United States, using uniform criteria for measurement. The software was developed and tested using hydropower information and data provided by the Southwestern Power Administration. It is a menu-driven program that allows the personal computer user to assign environmental attributes to potential hydropower sites, calculate development suitability factors for each site based on the environmental attributes present, and generate reports based on these suitability factors. This report describes the resource assessment results for the State of Illinois.
\end{abstract}




\section{CONTENTS}

ABSTRACT ……...............................................................................................................

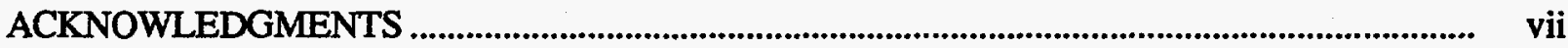

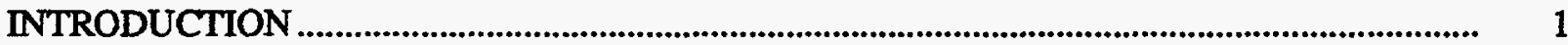

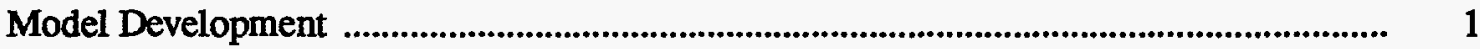

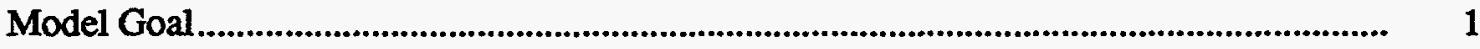

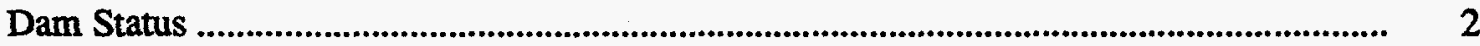

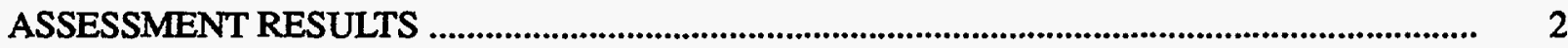

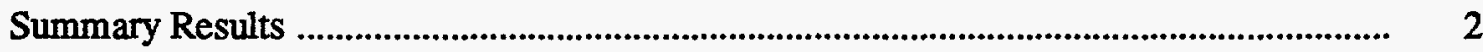

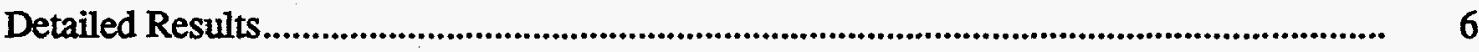

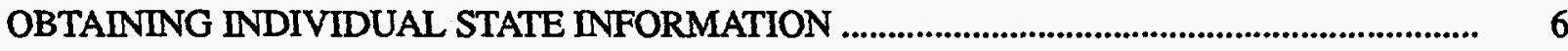

ADDITIONAL HYDROPOWER EVALUATION SOFTWARE INFORMATION ......................... 7

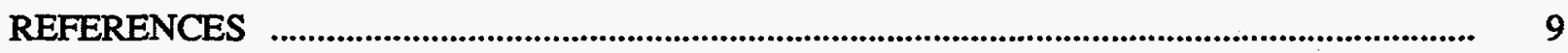

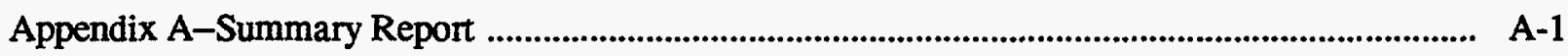

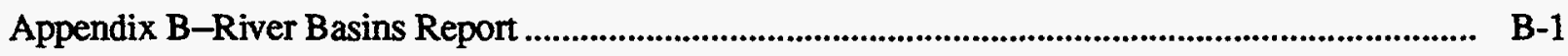

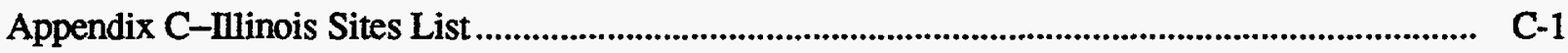

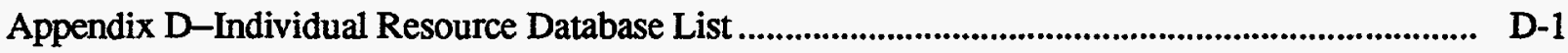

\section{FIGURES}

1. Number of sites, by capacity groups, with HES-modeled undeveloped hydropower potential .... 3

2. The nonmodeled and HES-modeled undeveloped hydropower potential ..................................... 3

3. The number of sites with undeveloped hydropower potential and the total megawatts of HES-modeled undeveloped hydropower potential.

4. The Dayton Dam on the Fox River is an example of a developed Illinois hydropower plant....... 4

5. Number of sites with undeveloped hydropower potential in the Ilinois river basins .................. 5

6. Megawatts of HES-modeled undeveloped hydropower potential in the Illinois river basins........ 5

\section{TABLES}

1. Undeveloped hydropower potential summaries for Illinois 


\section{ACKNOWLEDGMENTS}

The authors thank Peggy A. M. Brookshier, John V. Flynn, and Wayne Belgrave of the U.S. Department of Energy and Robert Dalton of the State of Illinois for their active participation and timely comments. 


\section{U.S. Hydropower Resource Assessment for Illinois}

\section{INTRODUCTION}

In June 1989, the U.S. Department of Energy initiated the development of a National Energy Strategy to identify the energy resources available to support the expanding demand for energy in the United States. Public hearings conducted as part of the strategy development process indicated that undeveloped hydropower resources were not well defined. As a result, the Department of Energy established an interagency Hydropower Resource Assessment Team to ascertain the undeveloped hydropower potential. In connection with these efforts by the Department of Energy, the Idaho National Engineering Laboratory designed the Hydropower Evaluation Software (HES), which has been used to perform a resource assessment of the undeveloped conventional hydropower potential in over 30 states. This report presents the results of the hydropower resource assessment for the State of Illinois. Undeveloped pumped storage hydropower potential is not included.

The HES was developed as a tool to measure undeveloped hydropower potential regionally or by state. The software is not intended to provide precise development factors for individual sites, but to provide regional or state totals. Because the software was developed as a generic measurement tool encompassing national issues, regional and state totals must be considered judiciously; various local issues may skew undeveloped hydropower potential totals. The information for the resource assessment was compiled from the Federal Energy Regulatory Commission's Hydroelectric Power Resources Assessment database and several other sources. Refer to DOE/ID-10338, the User's Manual (Francfort, Matthews, Rinehart 1991) for the specifics of the software and to DOE/ID10430.1, the Status Report (Conner, Francfort, Rinehart 1996) for an overview of all resource assessment activities to date.

\section{Model Development}

Hydropower Evaluation Software, both a probability-factor computer model and a database, is a menu-driven program that is intended to be user-friendly. Computer screens and report-generation capabilities were developed to meet the needs of users nationwide. The software uses environmental attribute data to generate an overall project environmental suitability factor (PESF) between 0.1 and 0.9 , where 0.9 indicates the highest likelihood of development and 0.1 indicates the lowest likelihood of development. The suitability factors are dependent on the unique environmental attributes of each potential site. They reflect the considerations that (a) environmental concerns can make a potential site unacceptable, prohibiting its development (for a suitability factor of 0.1 ), or (b) if there are no environmental concerns, there is no effect on the likelihood of site development (for a suitability factor of 0.9 ). A combination of attributes can result in a lower suitability factor because multiple environmental considerations would reduce the likelihood that a site may be developed to its physical potential.

\section{Model Goal}

The goal of the HES is to assemble an accurate resource database of all sites with undeveloped hydropower potential in the United States for use as a planning tool to determine the viable national hydropower potential. Undeveloped hydropower potential is not limited to the development of new sites; it also includes the development of additional hydropower-generating capacity at sites that currently have hydropower, but are not developed to their full potential. This undeveloped hydropower potential is a source of nonpolluting, renewable energy available to meet the growing power needs of the United States. The HES should help make this goal obtainable and ensure a set of uniform criteria for national assessment. 


\section{Dam Status}

The effects of environmental attributes vary by dam status. The dam status classifications used are as follows

$\mathrm{W}=$ Developed hydropower site with current power generation, but the total hydropower potential has not been fully developed. Only the undeveloped hydropower potential is discussed in this report.

$\mathrm{W} / \mathrm{O}=$ Developed site without current power generation. The site has some type of developed impoundment or diversion structure, but no developed hydropower generating capability.

$\mathrm{U}=$ Undeveloped site. The site does not have power generation capability nor a developed impoundment or diversion structure.

\section{ASSESSMENT RESULTS}

\section{Summary Results}

A total of 49 sites (Table 1) have been identified and assessed for their undeveloped hydropower potential. The HES results for individual site capacities range from 107 kilowatts $(\mathrm{kW})$ to 60 megawatts (MW). The majority of the sites (59\%) have potential capacities of greater than $1 \mathrm{MW}$, but less than $10 \mathrm{MW}$ (Figure 1).

The nonmodeled undeveloped hydropower potential total for Illinois was identified as 594 MW. The HES results lowers this estimate about $49 \%$ to $301 \mathrm{MW}$. The greatest reduction in undeveloped hydropower potential, by MW, occurs at sites with some type of structure in place, but no current generation of power present (without power category). These sites have an HES-modeled undeveloped hydropower potential of $242 \mathrm{MW}$, a $215 \mathrm{MW}$ reduction in the estimated undeveloped hydropower potential (Figure 2). The undeveloped sites have the greatest percentage decrease in modeled undeveloped potential capacity. The unadjusted potential is $58 \mathrm{MW}$, and the modeled capacity is $18 \mathrm{MW}$, a 69\% decrease in capacity (Figure 2). The number of sites does not change, only the identified undeveloped hydropower potential is reassessed (Figure 3).

The 49 identified sites are located within 9 major river basins and 1 minor river basin. The Dayton Project on the Fox River (Figure 4) illustrates a developed Illinois hydropower plant. The number of sites per major river basin ranges from 1 in the Meramec and Ohio Main Stream River Basins, to 22 in the Illinois River Basin (Figure 5). The Mississippi River Basin has the most undeveloped hydropower potential (110 MW) of the Illinois river basins (Figure 6). It should be noted that the Mississippi River Basin reports to having only 3 sites to develop in order to obtain the $110 \mathrm{MW}$ of undeveloped hydropower capacity.

Table 1. Undeveloped hydropower potential summaries for Illinois. The table contains the nonmodeled undeveloped nameplate potential and the HES-modeled undeveloped hydropower potential totals.

Nameplate potential HES-modeled potential

Number of projects $\quad$ (MW) (MW)

\begin{tabular}{lrrr} 
With Power & 9 & 80.1 & 41.4 \\
W/O Power & 35 & 456.7 & 242.0 \\
Undeveloped & 5 & 57.6 & 17.5 \\
State Total & 49 & 594.4 & 300.9 \\
\hline
\end{tabular}




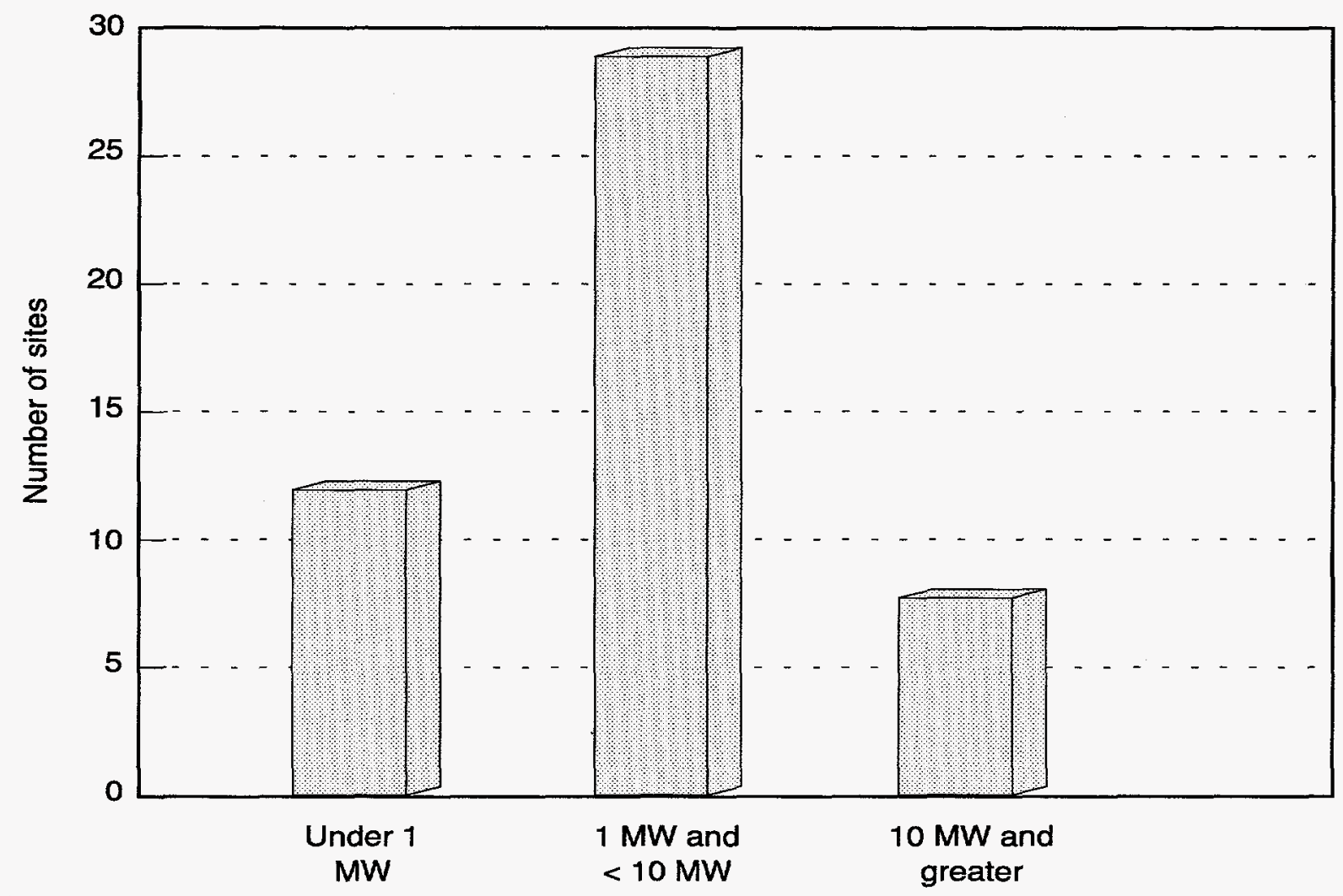

Figure 1. Number of sites, by capacity groups, with HES-modeled undeveloped hydropower potential.

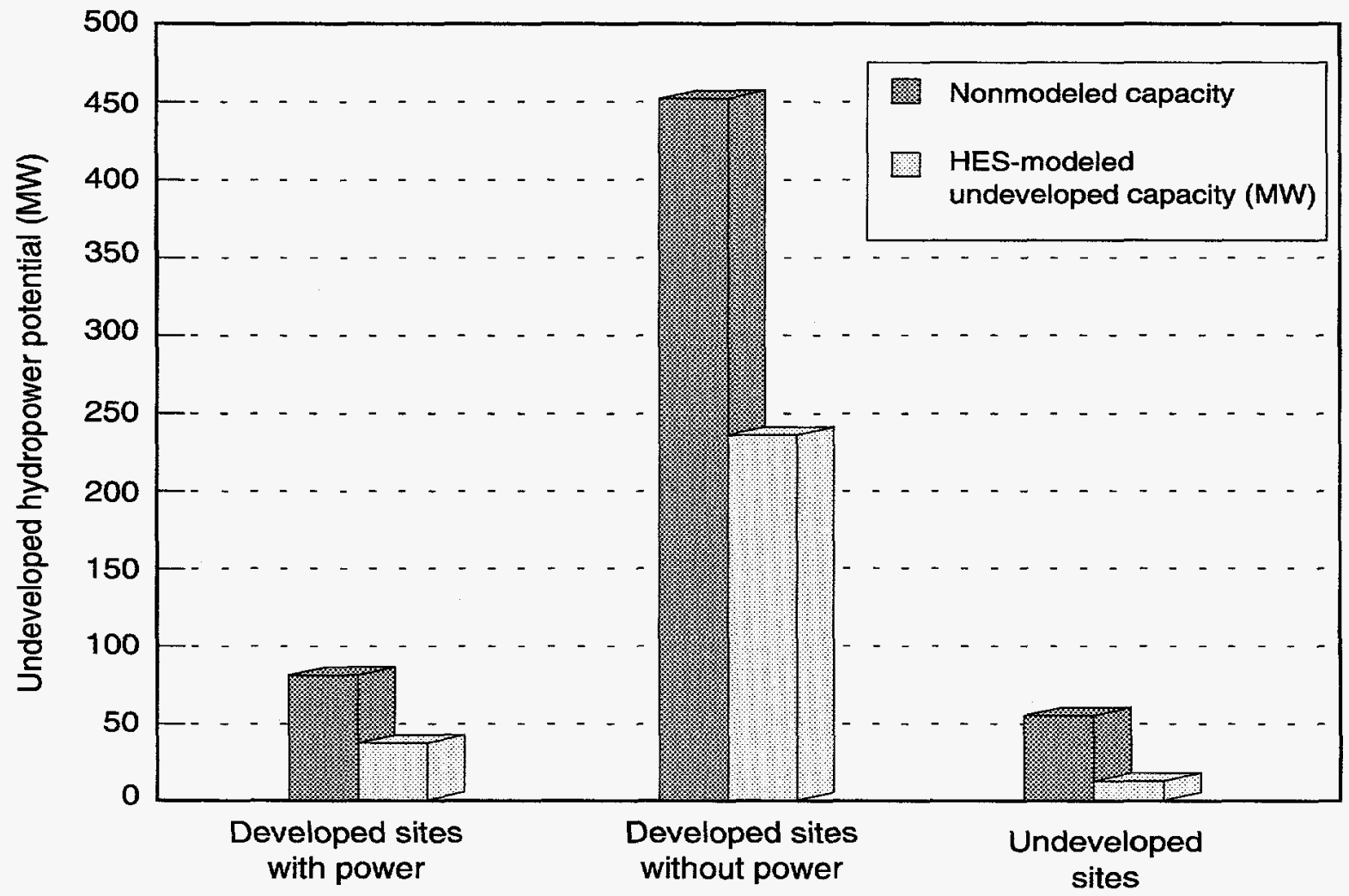

Figure 2. The nonmodeled and HES-modeled undeveloped hydropower potential. 


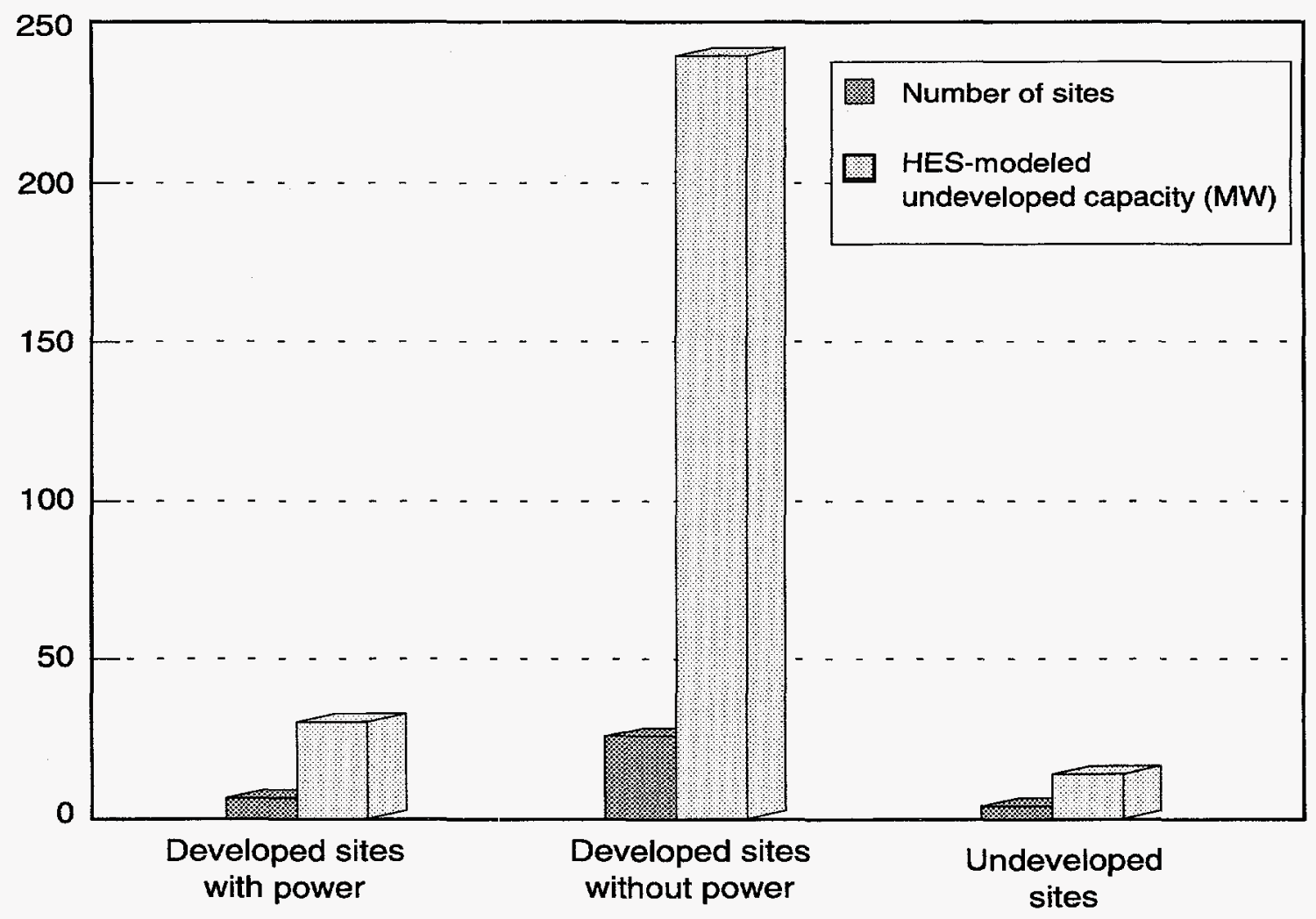

Figure 3. The number of sites with undeveloped hydropower potential and the total megawatts of HESmodeled undeveloped hydropower potential.

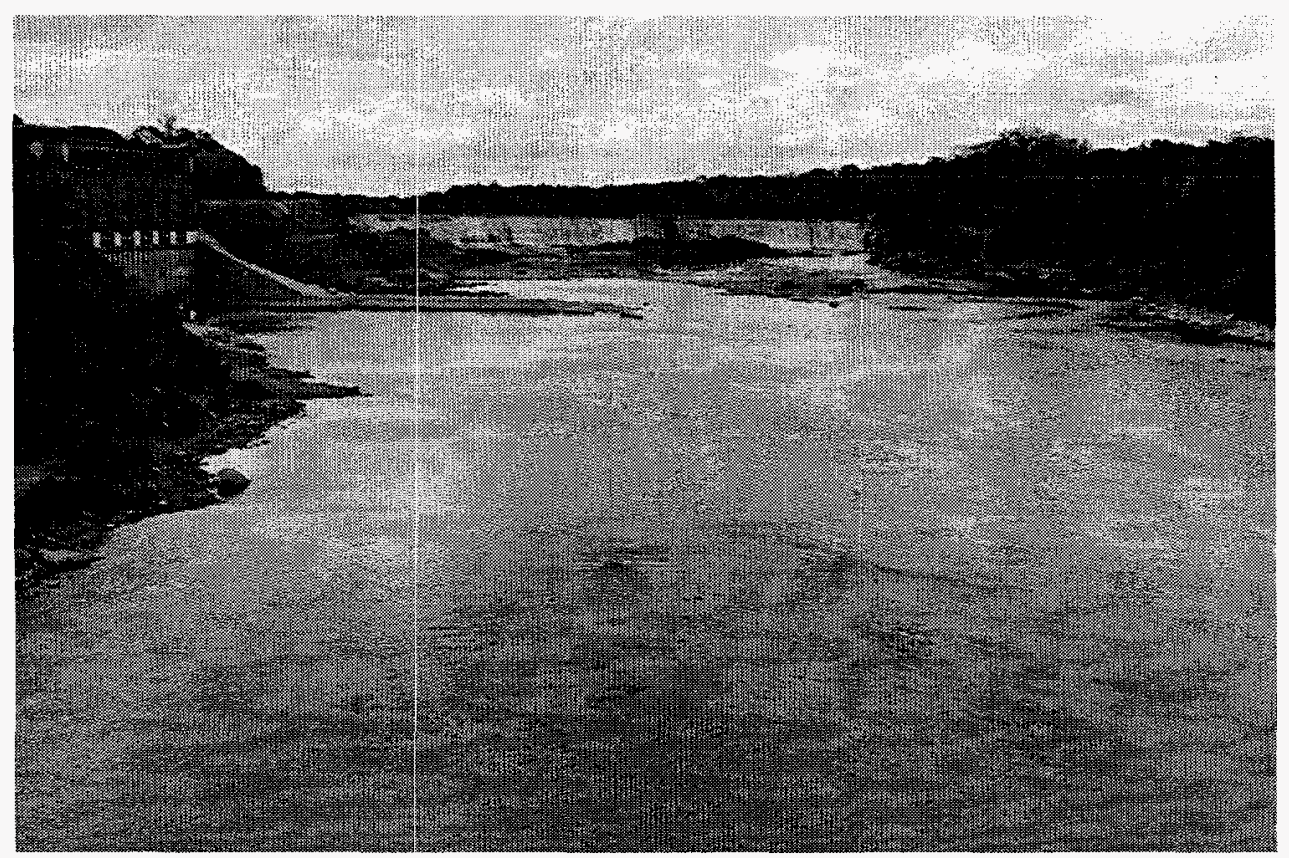

Figure 4. The Dayton Dam on the Fox River is an example of a developed Illinois hydropower plant. The photo shows the Dayton power plant on the left side and the Dayton Reservoir dam in the background. The Dayton plant (FERC \#2137) was constructed in the 1920s and has a nameplate rating of 3.6 MW. It has an additional undeveloped capacity of $630 \mathrm{~kW}$ at this 23 -foot structure. 


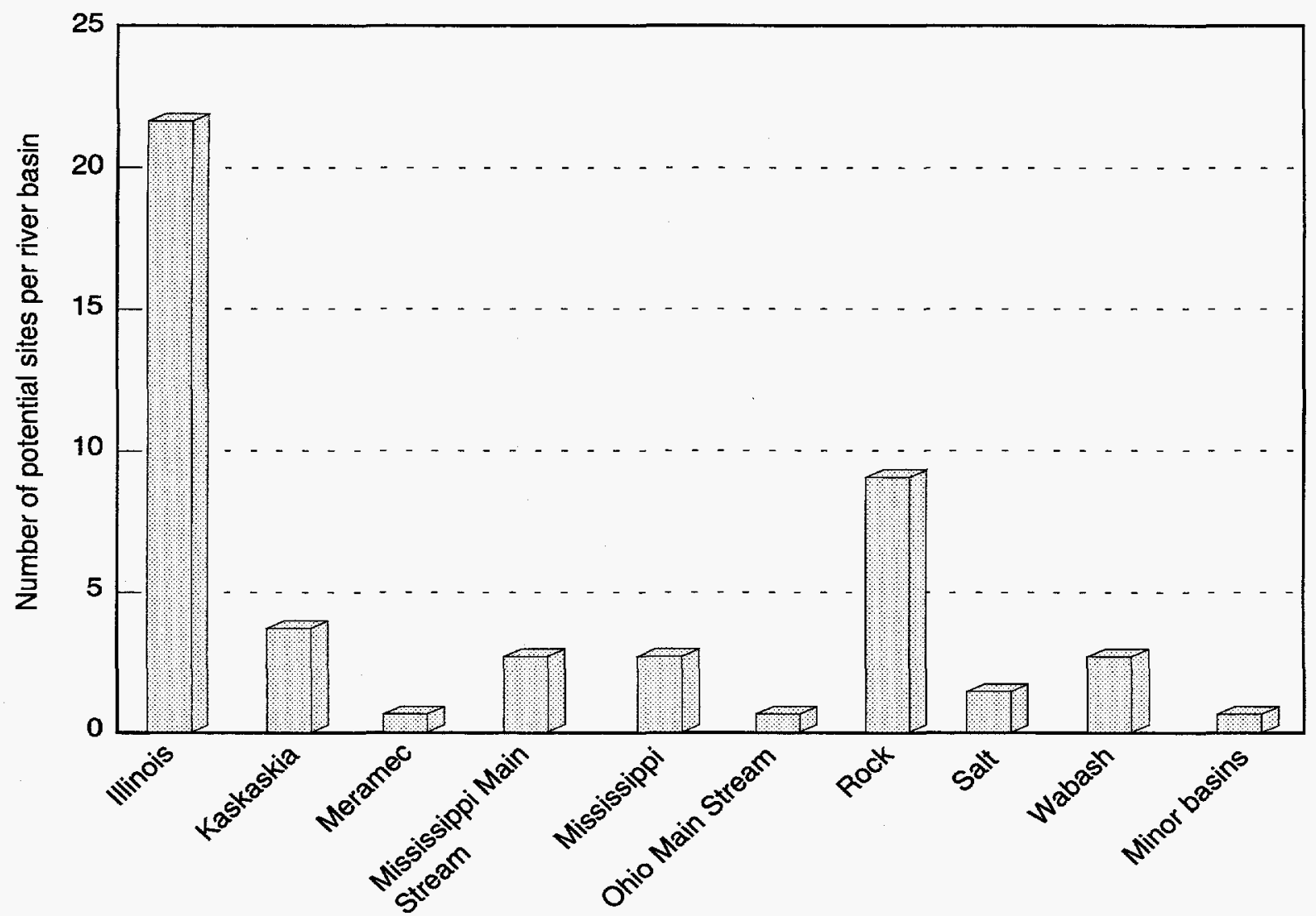

Figure 5. Number of sites with undeveloped hydropower potential in the Illinois river basins.

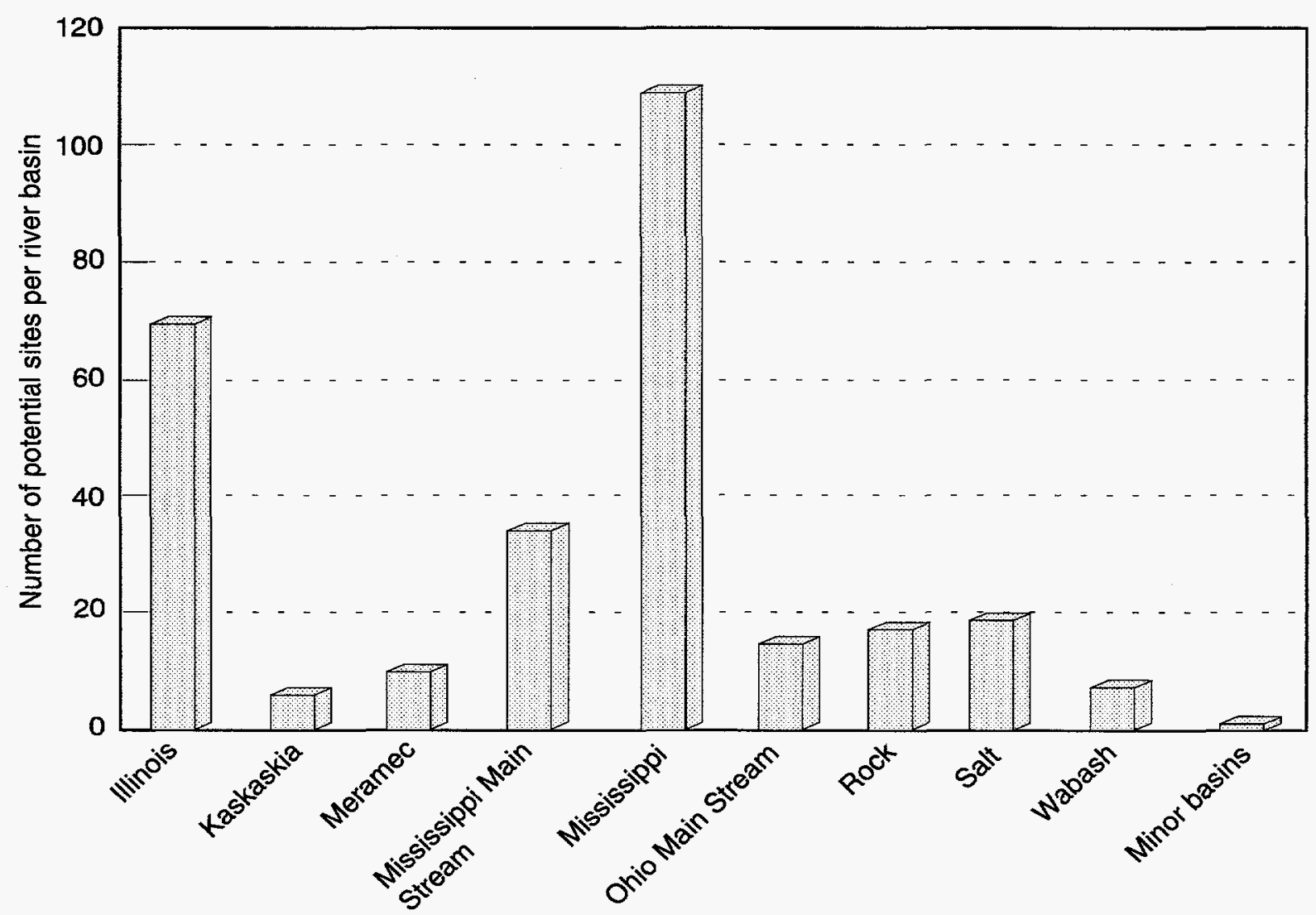

Figure 6. Megawatts of HES-modeled undeveloped hydropower potential in the Illinois river basins. 


\section{DETAILED RESULTS}

The appendices contain, in the form of HES-generated reports, detailed information about the undeveloped hydropower potential in Illinois. The appendices contain the following information:

Appendix A summarizes the undeveloped hydropower potential by dam status groups. The number of sites, nonmodeled undeveloped hydropower potential, and HES-modeled undeveloped hydropower potential is provided based on the dam status.

Appendix B provides the hydropower resource assessment by river basin, which includes the project number, project name, stream name, dam status, nonmodeled undeveloped hydropower potential, and the HES-modeled undeveloped hydropower potential. for each of the individual sites. Subtotals are provided for each river basin.

Appendix C provides the hyclropower resource assessment by FERC number, which includes the project numbers, plant name, stream name, if a site is Federally owned, nonmodeled undeveloped hydropower potential, and HESmodeled undeveloped hydropower potential. The sites are grouped by dam status.

Appendix D contains a resource database list for each of the 49 sites in Illinois. Information includes plant name, stream, state, county, river basin and owner names, project number, name plate and HES-modeled undeveloped hydropower potential, the unit and plant types, dam status, latitude, longitude, and the environmental factors that the HES uses to determine the project environmental suitability factor.

\section{OBTAINING INDIVIDUAL STATE INFORMATION}

Additional copies of the hydropower resource assessment results for individual states are available and can be obtained by writing or calling the authors or the National Technical Information Service (NTIS).
Telephone Orders-(703) 487-4650. NTIS sales desk and customer services are available between 8:30 a.m. and 5:00 p.m., EST.

Fax-(703) 321-8547. Customers may fax their orders to NTIS. These orders may be charged to a NTIS deposit account, American Express, VISA, or MasterCard.

Mail Orders-Mail orders should be sent to National Technical Information Service, Document Sales, 5285 Port Royal Road, Springfield, VA 22161. Call the sales desk for prices before placing an order.

Method of Payment-Customers may pay for reports (and other NTIS products and services) by (a) credit card (American Express, Visa or MasterCard); (b) check or money order on a United States bank payable to NTIS; (c) an NTIS deposit account; or, (d) by asking to be billed (add $\$ 7.50$ per order), United States, Canada, and Mexico, only.

Handling Fee-A $\$ 3.00$ handling fee per total order applies to orders from the United States, Canada, and Mexico. Handling charges do not apply to rush order service or pick-up orders.

Postage and Shipping-Orders are shipped first class mail, or equivalent, to addresses in the United States, Canada, and Mexico.

Order Turnaround Time-Orders for technical reports generally are shipped within 2 to 8 days of receipt. For faster service, NTIS offers rush order service.

Rush Order Service-Call 1-800-533-NTIS. In Virginia, Canada, and Mexico call (703) 4874700. For NTIS rush order service add $\$ 15.00$ per item. This guarantees that an order will be processed through NTIS within 24 hours of its receipt. These orders receive immediate, individual attention. The items ordered are delivered by first call mail. Call NTIS for information on rush order service for computer products.

For Help in Tracing an Order-Call (703) 487-4650 and request the customer service option. 


\section{ADDITIONAL HYDROPOWER EVALUATION SOFTWARE INFORMATION}

Additional information concerning the HES can be obtained by contacting Ben Rinehart or Jim Francfort at the addresses provided below. Copies of the software and the User's Manual may also be obtained from these individuals.

Ben Rinehart, Project Manager Idaho National Engineering Laboratory P.O. Box 1625, MS 3830

Idaho Falls, ID 83415-3830

(208) 526-1002
Jim Francfort

Idaho National Engineering Laboratory

P.O. Box 1625, MS 3830

Idaho Falls, ID 83415-3830

(208) 526-6787

Information concerning the State of Illinois' involvement with the resource assessment or about the identified sites may be obtained by contacting:

\section{Robert Dalton}

State of Illinois

Chief of the Downstate Regulatory Program

Division of Water Resources

Springfield, IL 62701

(217) $782-3863$ 


\section{REFERENCES}

Conner, A. M., J. E. Francfort, and B. N. Rinehart, 1996, Uniform Criteria for U.S. Hydropower Resource Assessment, Hydropower Evaluation Software Status Report-II, DOE/ID 10430.1, Idaho National Engineering Laboratory, Idaho Falls, Idaho.

Francfort, J. E., S. D. Matthews, and B. N. Rinehart, 1991, Hydropower Evaluation Software User's Manual, DOE/ID-10338, Idaho National Engineering Laboratory, Idaho Falls, Idaho. 
Appendix A

Summary Report 
$H Y D R O P O W E R$

state

Category

With Power

W/O Power

Undeveloped

STATE TOTAL

TOTALS

With Power

W/O Power

Undeveloped

GRAND TOTAL
C A P A C I T Y

Projects

9

35

5

49

9

35

5

49
DATE: $12 / 06 / 96$

$S U M M A R Y$
Name Plate

Capacity (KW)

HES Adjusted Capacity (KW)

$$
80065
$$

41395

456677

57600

241991.75

17525

594342

300911.75

80065

41395

456677

57600

241991.75

17525

594342

300911.75 


\section{Appendix B \\ River Basins Report}


Page No.

$12 / 06 / 96$

FERC

Number
1

HYDROPOWER RESOURCE ASSESSMENT BY RIVER BASIN

Dam Name Plate

Stat Rating (KW) PESF PESF * KW

** River Basin ILIINOIS RIVER BASIN

IL008 CUSTER PARK

KANKAKEE R

04031 STARVED ROCK I\&D

IILINOIS $\mathrm{R}$

IL003 DAYTON

EOX R

08632 KANKAKEE

KANKAKEE RIVER

SANITARY \& SHIP CANAI

IL004 WEDRON

FOX R

FOX R

02961 HOTEL BAKER

FOX R

11559 MARSEILLES I\&D

ILIINOIS R.

IL005 MARSEILIES

ILIINOIS R

IL006 MARSEILIES

ILLINOIS R

03943 DRESDEN ISLAND I\&D

ILIINOIS $R$

03944 BRANDON ROAD L\&D

DES PLAINES $R$

IL007 OLD STATE

KANKAKEE R

09828 WILMINGTON

KANKAKEE R

IL102 IAAGRANGE

ILIINOIS RIVER
U

W

W

W

wo

wo

$15000.00 \quad 0.25$

3750.00

W

$7600.00 \quad 0.50$

3800.00

315.00

$630.00 \quad 0.50$

$2250.00 \quad 0.75$

1687.50

$13500.00 \quad 0.50$

6750.00

พอ

wo

wo

wo

wo

WO

wo

wo

wo
4000.00

1012.50

$8000.00 \quad 0.50$

$1350.00 \quad 0.75$

$143.00 \quad 0.75$

$6840.00 \quad 0.50$

$2024.00 \quad 0.50$

$11000.00 \quad 0.50$

$10500.00 \quad 0.50$

5250.00

$6100.00 \quad 0.50$

$6600.00 \quad 0.90$

5940.00

$600.00 \quad 0.50$

300.00

$12160.00 \quad 0.75$

9120.00
3050.00 
Page No.

$12706 / 96$

2

HYDROPOWER RESOURCE ASSESSMENT BY RIVER BASIN

FERC

Number

ILI01 ELGIN, KIMBALI STREET

FOX RIVER

ILI03 YORKVILIE

FOX RIVER

ILI04 LAKE DECATUR

SANGAMON RIVER

ILI07 PEORIA

ILIINOIS RIVER

IL109 LAKE SPRINGFIELD SUGAR CREEK

11307 MARSEILLES HYDROELECTRIC ILIINOIS RIVER

** Subtotal **

* * River Basin KASKASKIA RIVER BASIN

09256 KASKASKIA I\&D KASKASKIA R

11214 CARIYIE

KASKASKIA R

11567 SHELBYVILLE

KASKASKIA R

ILI06 LARE LOU YAEGER

WEST FORK SHOAL CREEK

** Subtotal **

* * River Basin MERAMEC RIVER BASIN

ILOI5 MISSISSIPPI I\&D 27 MISSISSIPPI $R$

* * Subtotal **

* * River Basin MINOR RIVER BASINS

09051 REND LAAKE DAM

BIG MUDDY $R$
Dam Name Plate
Stat Rating (KW) PESF PESF * KW

wo

$600.00 \quad 0.75$

450.00

wo

$1400.00 \quad 0.50$

700.00

WO

1160.000 .75

870.00

WO

$8750.00 \quad 0.75$

6562.50

wo

$2850.00 \quad 0.75$

2137.50

wo

$10600.00 \quad 0.50$

5300.00

129657.00

71034.25

wo

$3200.00 \quad 0.50$

1600.00

wo

$4000.00 \quad 0.50$

2000.00

wo

$6100.00 \quad 0.50$

3050.00

wo

$740.00 \quad 0.75$

555.00

14040.00

7205.00

wo

$15340.00 \quad 0.75$

11505.00

15340.00

11505.00

wo

$1600.00 \quad 0.50$

800.00 
Page No.
$12 / 06 / 96$

HYDROPOWER RESOURCE ASSESSMENT BY RIVER BASIN

FERC

Number

\section{Plant Name/}

Stream

Dam Name Plate

Stat Rating (KW) PESF

PESF * KW

800.00

W

$19680.00 \quad 0.50$

9840.00

ILO14 MOLINE LED 15
SYLVAN SLOUGH, MISS R

** Subtotal **

19680.00

9840.00

** River Basin MISSISSIPPI MAIN STREAM

07105 MISSISSIPPI L\&D 15 MISSISSIPPI RIVER

W

wo

03862

MISSISSIPPI L\&D 14 MISSISSIPPI $\mathrm{R}$

* * Subtotal **

* * River Basin MISSISSIPPI RIVER

03246 MISSISSIPPI L\&D $26 R$ MISSISSIPPI RIVER

WO

52000.00

26000.00

no

$119430.00 \quad 0.50$

59715.00

** Subtotal * *

** River Basin MISSISSIPPI RIVER BASIN
11223 L\&D 24 HYDROELECTRIC MISSISSIPPI RIVER

11224 IED 25 HYDROELECTRIC MISSISSIPPI RIVER

** Subtotal * *

* * River Basin OHIO MAIN STREAM

07647 BROOKPORT (L\&D 52) OHIO $R$

WO

wo

119430.00

59715.00

$50000.00 \quad 0.50$

25000.00

$50000.00 \quad 0.50$

25000.00

100000.00

50000.00

WO

$34500.00 \quad 0.50$

17250.00

** Subtotal **

** River Basin ROCK RIVER BASIN

ILO09 BARSTOW ROCK R
U

34500.00

17250.00

$12500.00 \quad 0.50$

6250.00 


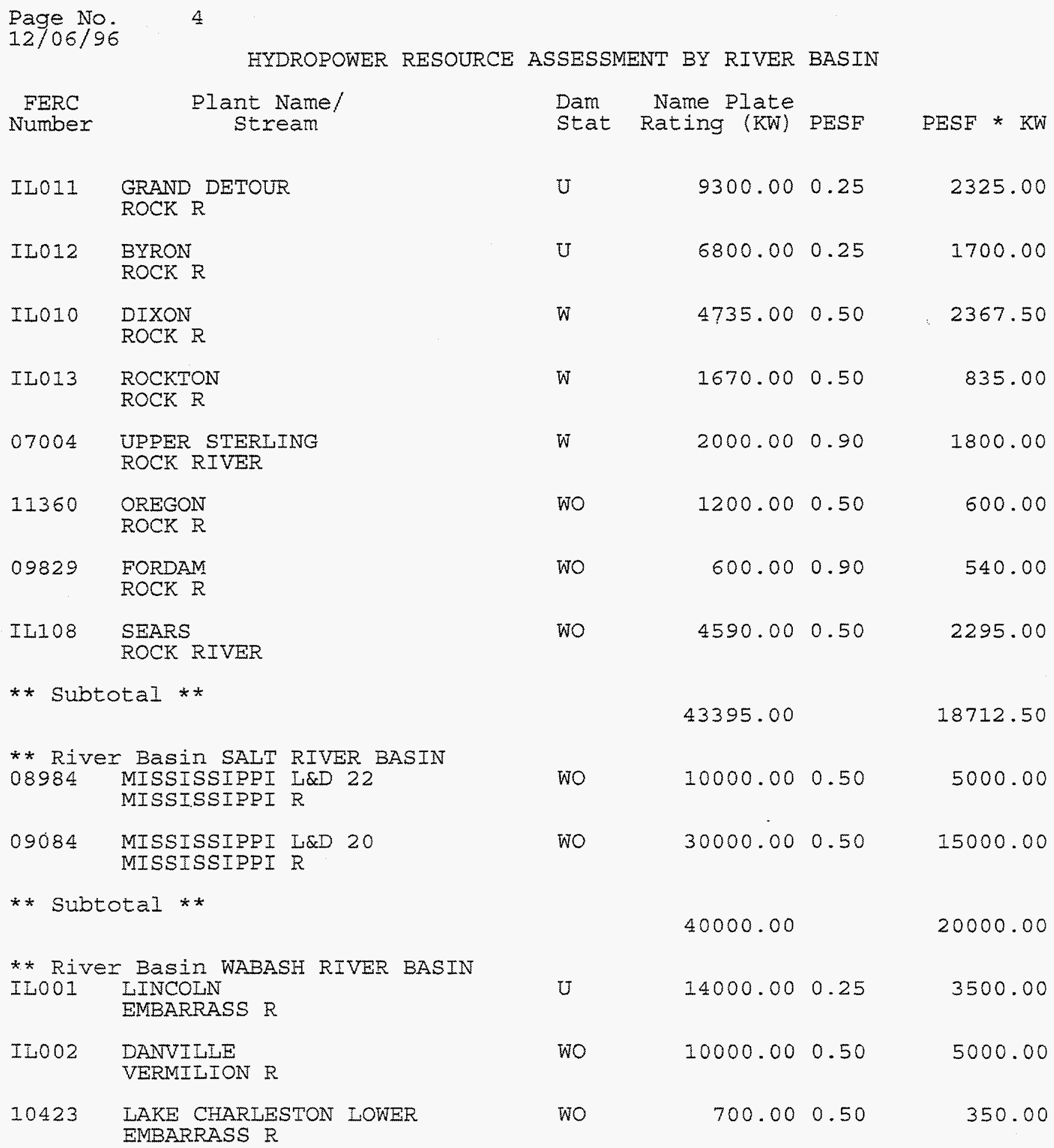


Page No.

5

$12 / 06 / 96$

HYDROPOWER RESOURCE ASSESSMENT BY RIVER BASIN

FERC

Number

$$
\begin{gathered}
\text { Plant Name/ } \\
\text { Stream }
\end{gathered}
$$

Dam Name plate

Stat Rating (KW) PESF
PESF * KW 8850.00 300911.75

** Subtotal **

*** Total ***

24700.00

594342.00 
Appendix C

Illinois Sites List 
Page No.

1

$12 / 06 / 96$

HYDROPOWER RESOURCE ASSESSMENT BY FERC NUMBER

Plant Name/

Stream

* * FERC Number II001

IINCOLN

EMBARRASS R

** Subtotal **

** FERC Number IL008

CUSTER PARK

KANKAKEE R

** Subtotal **

* * FERC Number IL009 BARSTOW

ROCK $\mathrm{R}$

* * Subtotal **

** FERC Number II011

GRAND DETOUR

ROCK R

** Subtotal **

** FERC Number IL012

BYRON

ROCK $R$

** Subtotal **

* * FERC Number 02866

LOCKPORT

SANITARY \& SHIP CANAL

** Subtotal * *

** FERC Number 04031

STARVED ROCK L\&D

ILIINOIS $\mathrm{R}$ $\begin{array}{ll}\text { Dam } & \text { Name Plate } \\ \text { ST } & \text { Rating (KW) PESF }\end{array}$

IL U

$14000.00 \quad 0.25$

14000.00

3500.00

IL $\quad U$

$15000.00 \quad 0.25$

3750.00

15000.00

3750.00

IL U

$12500.00 \quad 0.50$

6250.00

12500.00

6250.00

IL U

9300.00

0.25

2325.00

$$
9300.00
$$

2325.00

IL U

$$
6800.00 \quad 0.25
$$

1700.00

$$
6800.00
$$

1700.00

II $W$

$13500.00 \quad 0.50$

6750.00

13500.00

6750.00

IL $W$

$7600.00 \quad 0.50$
3800.00 
Page No.

$12 / 06 / 96$

HYDROPOWER RESOURCE ASSESSMENT BY FERC NUMBER

$$
\begin{gathered}
\text { Plant Name/ } \\
\text { Stream }
\end{gathered}
$$

** Subtotal **

** FERC Number 07004

UPPER STERLING

ROCK RIVER

* * Subtotal * *

* * FERC Number 07105

MISSISSIPPI L\&D 15

MISSISSIPPI RIVER

** Subtotal **

** FERC Number 08632 KANKAKEE

KANKAKEE RIVER

* * Subtotal * *

* * FERC Number IL003 DAYTON

FOX R

** Subtotal **

** FERC Number IL010 DIXON ROCK $R$

* * Subtotal * *

* * FERC Number IL013 ROCKTON

ROCK R

** Subtotal **

$\begin{array}{lll}\text { Dam } & \text { Name Plate } & \\ \text { ST Stat } & \text { Rating (KW) PESF } & \text { PESF * } \mathrm{KW}\end{array}$

IL $\quad W$

7600.00

3800.00

1800.00

2000.00

1800.00

IL $W$

$28000.00 \quad 0.50$

14000.00

28000.00

14000.00

II $\quad W$

$2250.00 \quad 0.75$

1687.50

2250.00

1687.50

IL W

$630.00 \quad 0.50$

315.00

630.00

315.00

IL W

$4735.00 \quad 0.50$

2367.50

4735.00

2367.50

II $W$

$1670.00 \quad 0.50$

835.00

1670.00

835.00 
Page No.

3

$12 / 06 / 96$

HYDROPOWER RESOURCE ASSESSMENT BY FERC NUMBER

\section{Plant Name/ \\ Stream}

* * FERC Number IL014

MOLINE ILD 15

SYLVAN SLOUGH, MISS R

* * Subtotal * *

** FERC Number 02961

HOTEL BAKER

FOX R

** Subtotal **

** FERC Number 03246

MISSISSIPPI L\&D 26R

MISSISSIPPI RIVER

* * Subtotal **

* * FERC Number 03862

MISSISSIPPI I\&D 14

MISSISSIPPI R

* * Subtotal **

* * FERC Number 03943

DRESDEN ISLAND L\&D

ILIINOIS $R$

* * Subtotal **

* * FERC Number 03944

BRANDON ROAD L\&D

DES PLAINES R

** Subtotal **

** FERC Number 07647

BROOKPORT (L\&D 52)

OHIO $R$

\section{Dam Name Plate \\ ST stat \\ Rating (KW) PESF}

PESF * KW

II W

$19680.00 \quad 0.50$

9840.00

19680.00

9840.00

II WO

$143.00 \quad 0.75$

107.25

143.00

107.25

II WO

$119430.00 \quad 0.50$

59715.00

119430.00

59715.00

IL Wo

$24000.00 \quad 0.50$

12000.00

24000.00

12000.00

IL WO

$10500.00 \quad 0.50$

5250.00

10500.00

5250.00

IL WO

$6100.00 \quad 0.50$

3050.00

6100.00

3050.00

II wo

$34500.00 \quad 0.50$

17250.00 
Page No.

$12706 / 96$

4

HYDROPOWER RESSOURCE ASSESSMENT BY FERC NUMBER

$$
\begin{gathered}
\text { Plant Name/ } \\
\text { Stream }
\end{gathered}
$$

** Subtotal **

** FERC Number 08984

MISSISSIPPI L\&D 22

MISSISSIPPI R

* * Subtotal **

* * FERC Number 09051

REND LAAKE DAM

BIG MUDDY $R$

** Subtotal **

** FERC Number 09084

MISSISSIPPI L\&D 20

MISSISSIPPI R

* * Subtotal **

** FERC Number 09256

KASKASKIA I\&D

KASKASKIA $R$

* * Subtotal **

** FERC Number 09828

WILMINGTON

KANKAKEE R

** Subtotal **

** FERC Number 09829

FORDAM

ROCK R

** Subtotal **

\section{Dam Name Plate \\ ST Stat Rating (KW) PESF}

34500.00

17250.00

IL WO

$10000.00 \quad 0.50$

5000.00

10000.00

5000.00

IL WO

1600.00

0.50

800.00

1600.00

800.00

IL wo

$30000.00 \quad 0.50$

15000.00

30000.00

15000.00

II WO

3200.00

0.50

1600.00

3200.00

1600.00

II WO

$600.00 \quad 0.50$

300.00

600.00

300.00

II WO

$600.00 \quad 0.90$

540.00

600.00 
5

HYDROPOWER RESOURCE ASSESSMENT BY FERC NUMBER

Plant Name/ Stream

* * EERC Number 10423

LAKE CHARIESTON LOWER EMBARRASS R

** Subtotal **

* * FERC Number 11214

CARLYLE

KASKASKIA $R$

** Subtotal **

* * FERC Number 11223

L\&D 24 HYDROELECTRIC

MISSISSIPPI RIVER

** Subtotal **

* * FERC Number 11224

L\&D 25 HYDROELECTRIC

MISSISSIPPI RIVER

* * Subtotal * *

** FERC Number 11307

MARSEILIES HYDROELECTRIC

ILIINOIS RIVER

** Subtotal * *

* FERC Number 11360

OREGON

ROCK R

$* *$ Subtotal **

* * FERC Number 11380

GENEVA

FOX R

\section{Dam Name Plate \\ ST Stat \\ Rating (KW) PESF}

IL WO

II wO

$4000.00 \quad 0.50$

IL WO

II WO

IL wo

IL WO

IL Wo
$700.00 \quad 0.50$

700.00

4000.00

$50000.00 \quad 0.50$

50000.00

PESE * KW

350.00

350.00

2000.00

2000.00

25000.00

25000.00

$50000.00 \quad 0.50$

25000.00

50000.00

25000.00

5300.00

10600.00

5300.00

600.00

1200.00

600.00

1012.50 
Page No.

6

$12 / 06 / 96$

HYDROPOWER RESOURCE ASSESSMENT BY FERC NUMBER

Name Plate
Plant Name/

Stream

* * Subtotal **

* * FERC Number 11559

MARSEILIES I\&D

ILIINOIS R

* * Subtotal **

** FERC Number 11567

SHELBYVILLE

KASKASKIA $R$

* * Subtotal ** DANVILLE

VERMILION R
* * FERC Number IL002

ST Stat

PESF * KW

1012.50

IL wo

1350.00

$6840.00 \quad 0.50$

3420.00

6840.00

3420.00

IL wo

6100.00

0.50

3050.00

6100.00

3050.00

IL WO

$10000.00 \quad 0.50$

5000.00

10000.00

5000.00

IL WO

$8000.00 \quad 0.50$

4000.00

WEDRON

FOX R

** Subtotal **

* * EERC Number IL005 MARSEILIES

ILIINOIS $\mathrm{R}$

IL wo

8000.00

4000.00

$2024.00 \quad 0.50$

1012.00

* * SubtotaI **

* * EERC Number IL006

MARSEILIES

ILIINOIS $\mathrm{R}$

II WO

2024.00

1012.00

* * Subtotal **
$11000.00 \quad 0.50$

11000.00
5500.00

5500.00 
Page No.

7

$12 / 06 / 96$

HYDROPOWER RESOURCE ASSESSMENT BY FERC NUMBER

\section{Plant Name/ stream}

* * FERC Number IL007

OLD STATE

KANKAKEE R

* * Subtotal * *

** FERC Number IL015

MISSISSIPPI L\&D 27

MISSISSIPPI R

** Subtotal **

* * FERC Number IL101

ELGIN, KIMBALL STREET

FOX RIVER

* * Subtotal **

* * FERC Number ILIO2

LAGRANGE

ILIINOIS RIVER

** Subtotal **

** FERC Number ILI03

YORKVILIEE

FOX RIVER

** Subtotal **

** FERC Number ILI04

LAKE DECATUR

SANGAMON RIVER

** Subtotal **

* * FERC Number IL106

LAKE LOU YAEGER

WEST FORK SHOAL CREEK

\begin{abstract}
Dam Name Plate
$\mathrm{ST}$ Stat Rating (KW) PESF PESF * KW
\end{abstract}

IL WO

$$
6600.00 \quad 0.90
$$

5940.00

$$
6600.00
$$

IL WO

$$
15340.00 \quad 0.75
$$

11505.00

$$
15340.00
$$

IL WO

$$
600.00 \quad 0.75
$$

450.00

$$
600.00
$$

450.00

II WO

$12160.00 \quad 0.75$

9120.00

12160.00

9120.00

IL WO

$$
1400.00 \quad 0.50
$$

700.00

1400.00

700.00

IL Wo

$1160.00 \quad 0.75$

870.00

1160.00

870.00

IL WO

$740.00 \quad 0.75$

555.00 
8

$12706 / 96$

HYDROPOWER RJSOURCE ASSESSMENT BY FERC NUMBER

$$
\begin{gathered}
\text { Plant Name/ } \\
\text { Stream }
\end{gathered}
$$

** Subtotal **

* * FERC Number IL107

PEORIA

ILLINOIS RIVER

* * Subtotal **

* * FERC Number II 108 SEARS

ROCK RIVER

** Subtotal **

* * FERC Number IL109

IAKE SPRINGFIELD

SUGAR CREEK

** Subtotal * *

\begin{tabular}{|c|c|c|c|c|}
\hline \multirow[t]{2}{*}{$S T$} & \multirow{2}{*}{$\begin{array}{l}\text { Dam } \\
\text { Stat }\end{array}$} & $\begin{array}{l}\text { Name Plate } \\
\text { Rating }(\mathrm{KW})\end{array}$ & PESF & $\mathrm{PESE}$ * KW \\
\hline & & 740.00 & & 555.00 \\
\hline \multirow[t]{2}{*}{ IL } & Wo & 8750.00 & 0.75 & 6562.50 \\
\hline & & 8750.00 & & 6562.50 \\
\hline \multirow[t]{2}{*}{$I I$} & wo & 4590.00 & 0.50 & 2295.00 \\
\hline & & 4590.00 & & 2295.00 \\
\hline$I$ & Wo & 2850.00 & 0.75 & 2137.50 \\
\hline & & 2850.00 & & 2137.50 \\
\hline & & 594342.00 & & 300911.75 \\
\hline
\end{tabular}

*** Total *** 
Appendix D

Individual Resource Database List 

R E S O U R C E
D A $T A B A S E$
L I S T I N G

DATE: $12 / 06 / 96$

PAGE NO: 1

FERC

Number

Plant Name

02866 LOCKPORT

county Name

WILI

Stream

State

Name

SANITARY \& SHIP CANAL

IL

River Basin

ILIINOIS RIVER BASIN

$\begin{array}{cc}\text { Class } & \text { Owner Name } \\ \text { M } & \text { METRO WATER RECLAM DIST OF CHICAGO }\end{array}$

\begin{tabular}{|c|c|c|c|c|}
\hline $\begin{array}{l}\text { Name Plate } \\
\text { Rating (KW) }\end{array}$ & PESF & PESF* KW & $\begin{array}{l}\text { Annual Energy } \\
\text { Rating (MWh) }\end{array}$ & $\begin{array}{l}\text { PESF Annual } \\
\text { Energy Rating (MWh) }\end{array}$ \\
\hline 13500.00 & 0.50 & 6750.00 & 59130.00 & 29565 \\
\hline
\end{tabular}

$\begin{array}{lllll}\text { Unit } & \text { Plant } & \text { Project } & \text { Dam } & \text { Latitude } 4134.00 \\ \text { Type } & \text { Type } & \text { Status } & \text { Status } & \text { Longitude } 8805.00\end{array}$

ROR W

\section{Factor}

Wild/Scenic Protection

wild/Scenic Tributary or Upstream/Downstream wild/Scenic Location Cultural value

Fish Presence Value

Geologic Value

Historic Value

other Value

Recreation value

Scenic Value
Exist Prob

-

0.90

0.90

0.90

0.90

0.90

0.90

$Y \quad 0.75$

$Y \quad 0.75$

0.95
Factor

Exist Prob

Wildlife Value

Threatened/Endangered Fish

Threatened/Endangered Wildlife $\mathrm{Y}$

Federal Land Code 103

Federal Land Code 104

Federal Land Code 105

Federal Land Code 106

Federal Land Code 107

Federal Land Code 108

Federal Land Code 198
0.90

0.90

0.75

0.90

0.90

0.90

0.90

0.90

0.90

0.90 
DATE : $12 / 06 / 96$

PAGE NO: 2

FERC

Number

Plant Name

Stream

State

02961

HOTEL BAKER

FOX R

Name

County Name

River Basin

KANE

IILINOIS RIVER BASIN

Class Owner Name

I HOTEL BAKER

Name Plate

Rating ( $\mathrm{KW}$ )

PESF

Annual Energy

143.00

$0.75 \quad 107.25$

1200.00

IL

ESF Annua] Energy Rating (MWh)

$\begin{array}{ccccc}\text { Unit } & \text { Plant } & \text { Project } & \text { Dam } & \text { Latitude } \\ \text { Type } & \text { Type } & \text { Status } & \text { Status } & \text { Longitude } 8819.00 \\ & \text { ROR } & \mathrm{XX} & \text { WO } & \end{array}$

Factor

Exist Prob

Factor

Exist Prob

Wild/Scenic Protection

Wild/Scenic Tributary or Upstream/Downstream

Wild/Scenic Location

Cultural Value

Fish Presence Value

Geologic Value

Historic Value

other Value

Recreation Value

-

Factor

900

Scenic Value

0.90 Wildlife Value

Threatened/Endangered Fish

Threatened/Endangered Wildlife

0.90 Federal Land Code 103

0.90 Federal Land Code 104

0.90 Federal Land Code 105

0.90 Federal Land Code 106

0.90 Federal Iand Code 107

0.90 Federal Land Code 108

$\mathrm{Y}$

0.90
0.90

0.90

0.90

0.90

0.90

0.90

0.90

0.90

0.90

0.90 
RESOURCE DA TA B A S E I I S T I N G

DATE : $12 / 06 / 96$

PAGE NO: 3

FERC

Number

Plant Name

Stream

State

03246 MISSISSIPPI L\&D $26 \mathrm{R}$

MISSISSIPPI RIVER

Name

County Name

River Basin

MADISON

MISSISSIPPI RIVER

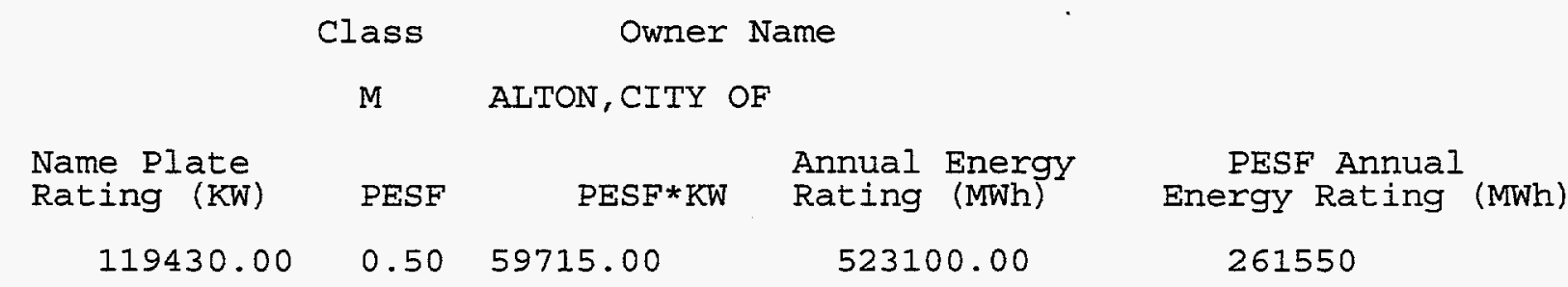

\begin{tabular}{|c|c|c|c|c|}
\hline $\begin{array}{l}\text { Unit } \\
\text { Type }\end{array}$ & $\begin{array}{l}\text { Plant } \\
\text { Type }\end{array}$ & $\begin{array}{l}\text { Project } \\
\text { status }\end{array}$ & $\begin{array}{l}\text { Dam } \\
\text { status }\end{array}$ & $\begin{array}{l}\text { Latitude } \\
\text { Longitude }\end{array}$ \\
\hline
\end{tabular}

ROR WO

Factor

Wild/Scenic Protection

Wild/Scenic Tributary or Upstream/Downstream wild/Scenic Location Cultural Value

Fish Presence Value

Geologic Value

Historic Value

other Value

Recreation Value

Scenic Value
Exist Prob

Factor

Exist Prob

0.90 Wildlife Value Threatened/Endangered Fish Y

0.90 Threatened/Endangered Wildlife

Federal Land Code 103

0.90 Federal Land Code 104

0.90 Federal Land Code 105

0.90 Federal Land Code 106

0.90 Federal Land Code 107

Y 0.75 Federal Land Code 108

0.90

0.90
II 

R E S O U R C E
$D A T A B A S E$
I I S T I N G

DATE : $12 / 06 / 96$

PAGE NO: 4

FERC

Number

Plant Name

Stream

State

03862

MISSISSIPPI L\&D 14

MISSISSIPPI $\mathrm{R}$

IL

County Name

ROCK ISLAND

Class

Owner Name

M LECIAIRE, CITY OF

Name Plate Rating (KW)

24000.00

PESF

$\mathrm{PESF} * \mathrm{KW}$

Annual Energy Rating (MWh)

$0.50 \quad 12000.00$

156000.00

Dam

Status

Latitude Longitude

4134.00

Type

Plant
Type

Project

ROR

MA

wo
PESF Annual Energy Rating (MWh) 78000
Factor

Wild/Scenic Protection Wild/Scenic Tributary or Upstream/Downstream wild/Scenic Location Cultural Value Fish Presence Value Geologic Value Historic Value other Value Recreation value Scenic Value
Exist Prob

Wildlife Value Threatened/Endangered Fish Threatened/Endangered Wildilfe $Y$

0.90

0.90

Federal Land Code 103

Federal Irand Code 104

0.90 Federal Land Code 105

0.90 Federal Land Code 106

0.90 Federal Land Code 107

Y $\quad 0.75$

0.90

0.90

Federal Land Code 108

Federal Land Code 198

Exist Prob

0.90

0.75

0.75

0.90

0.90

0.90

0.90

0.90

0.90

0.90 
DATE : $12 / 06 / 96$

PAGE NO: 5

FERC

Number

Plant Name

03943 DRESDEN ISIAAND L\&D

County Name

GRUNDY
Stream

ILIINOIS R

River Basin

ILIINOIS RIVER BASIN
State

Name

IL

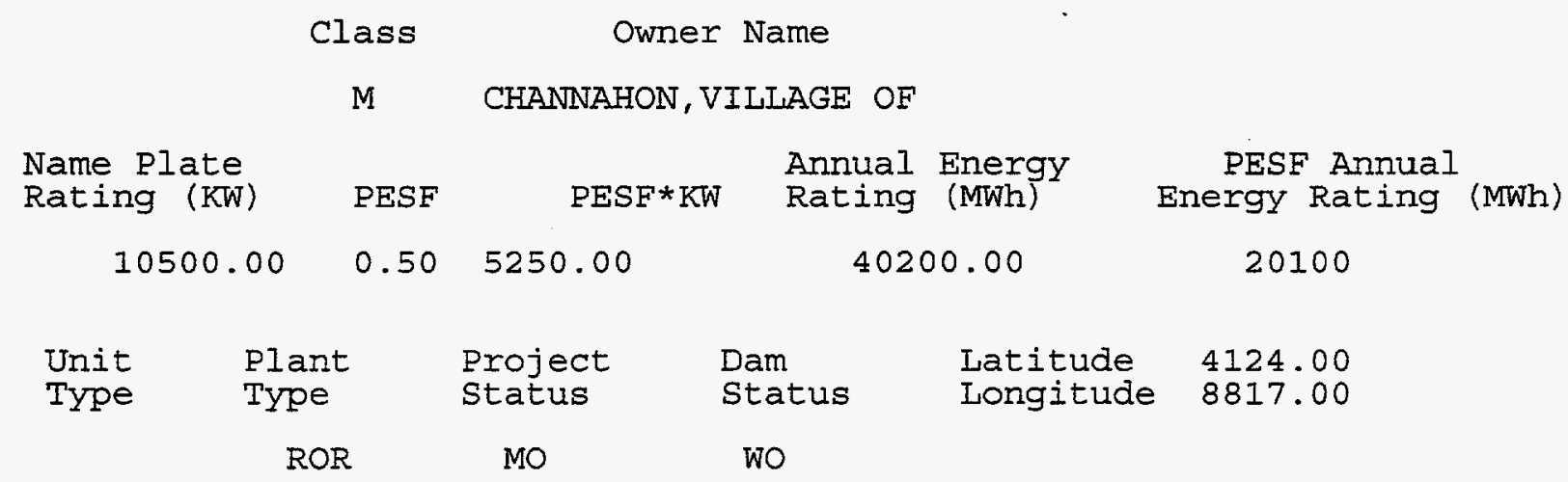

Factor

wild/Scenic Protection

Wild/Scenic Tributary or Upstream/Downstream wild/Scenic Location

Cultural value

Fish Presence Value

Geologic Value

Historic value

Other Value

Recreation value

Scenic Value
Exist Prob

0.90

Wildlife Value

Threatened/Endangered Fish Threatened/Endangered Wildlife

0.90

Federal Land Code 103

0.90 Federal Land Code 104

0.90 Federal Luand Code 105

0.90 Federal Land Code 106

0.90 Federal Land Code 107

$\begin{array}{ll}0.75 & \text { Federal Land Code } 108 \\ 0.75 & \text { Federal Land Code } 198\end{array}$

$\begin{array}{ll}Y & 0.75 \\ Y & 0.75\end{array}$
Exist Prob

0.90

0.75

0.90

0.90

0.75

0.90

0.90

0.90

0.90

0.90 
RESOURC E D A T A B A S E L I S T I N G

DATE : $12 / 06 / 96$

PAGE NO: 6

FERC

Number

Plant Name

Stream

State

03944

BRANDON ROAD L\&D

DES PLAINES $R$

II

County Name

WILL
River Basin

ILLINOIS RIVER BASIN

Class Owner Name

M ROCKDAIE, VILLAGE OF

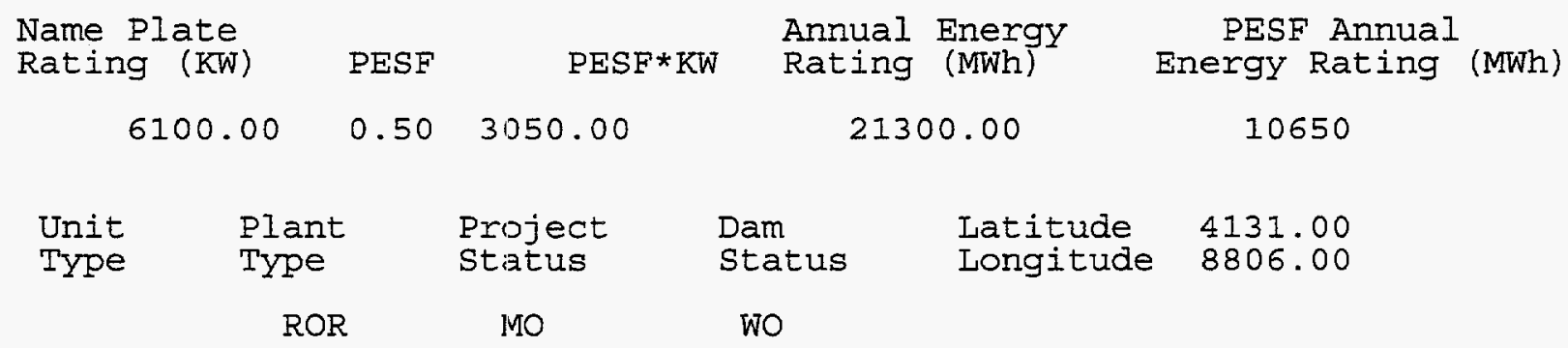

Factor

Exist Prob

Factor

Exist Prob

Wild/Scenic Protection

Wild/Scenic Tributary or

Upstream/Downstream

0.90 Wildlife Value

Threatened/Endangered Fish 0.90

Wild/Scenic Location

Threatened/Endangered

0.90 Federal Land Code 103

0.90 Federal Iuand Code 104

0.90 Federal Land Code 105

Wildlife

0.90

Cultural Value

Fish Presence Value

Geologic Value

0.90 Federal Land Code 106

0.90 Federal Land Code 107

Historic Value

other Value

Recreation value

0.75

Federal Land Code 107

0.90 Federal Land Code 198

0.90

$Y$

0.75

0.90

0.90

0.90

0.90

0.90

0.90 

R E S O U R C E
$D A$ T A A S E
L I S T I N G

DATE: $12 / 06 / 96$

PAGE NO: 7

FERC

Number

Plant Name

Stream

State

04031 STARVED ROCK L\&D

ILIINOIS $R$

Name

County Name

River Basin

ILA SALLE

ILLINOIS RIVER BASIN

Class Owner Name

M PERU, CITY OF

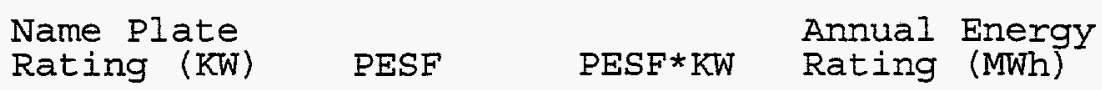

7600.00

$0.50 \quad 3800.00$

50330.00

PESF Annual Energy Rating (MWh)

25165

$\begin{array}{ccccc}\text { Unit } & \text { Plant } & \text { Project } & \text { Dam } & \text { Latitude } \\ \text { Type } & \text { Type } & \text { Status } & \text { Status } & \text { Longitude } 8859.00 \\ & \text { ROR } & \text { MO } & \text { W } & \end{array}$

Factor

Exist Prob

Factor

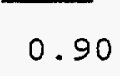

Wild/Scenic Protection

wild/Scenic Tributary or Upstream/Downstream wild/scenic Location Cultural value

Fish Presence Value

Geologic Value

Historic Value

other value

Recreation Value

Scenic Value
Exist Prob

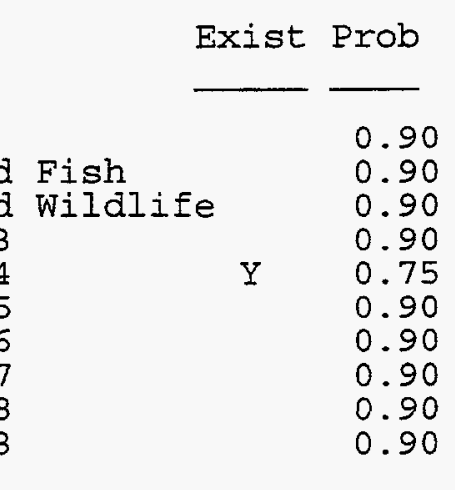

Wildife value Threatened/Endangered Fish Threatened/Endangered Wildife Federal Land Code 103

0.90 Federal Land Code 104

0.90 Federal Land Code 105

0.90 Federal Land Code 106

0.90 Federal Land Code 107

$Y \quad 0.75$ Federal Land Code 108

Y 0.75 Federal Land Code 198
IL 

R E S O U R
C E
D A T A B A S E
L I S T I N G

DATE : $12 / 06 / 96$

PAGE NO: 8

FERC

Number

Plant Name

Stream

State

Name

07004 UPPER STERIING

ROCK RIVER

II

County Name

River Basin

WHITESIDE

ROCK RIVER BASIN

Class Owner Name

M CITY OF ROCK FALLS

Name Plate

Rating (KW)

PESF

Annual Energy

PESF Annual

2000.00

$0.90 \quad 1800.00$

8760.00

Energy Rating (MWh)

Unit Plant

Type

Type

Project

status

ROR

Factor

Wild/Scenic Protection

Wild/Scenic Tributary or Upstream/Downstream

wild/Scenic Location

Cultural Value

Fish Presence Value

Geologic Value

Historic Value

other Value

Recreation Value

Scenic Value
Exist Prob

Prob

0.90

Wildlife Value

Threatened/Endangered Fish

Threatened/Endangered Wildlife

0.90 Federal Land Code 103

0.90 Federal Land Code 104

0.90 Federal Land Code 105

0.90 Federal Iuand Code 106

0.90 Federal Land Code 107

0.90 Federal Land Code 108

0.90

0.90
7884

4147.00

8941.00
Exist Prob

0.90

0.90

0.90

0.90

0.90

0.90

0.90

0.90

0.90

0.90 
DATE : $12 / 06 / 96$

PAGE NO: 9

FERC

Number

Plant Name

Stream

State

07105

MISSISSIPPI L\&D 15

MISSISSIPPI RIVER

Name

07105

County Name

River Basin

ROCK ISIAND

MISSISSIPPI MAIN STREAM

$\begin{array}{cc}\text { Class } & \text { Owner Name } \\ \text { P } & \text { DAVENPORT HYDRO ASSOCIATES }\end{array}$

\begin{tabular}{cccccc}
$\begin{array}{l}\text { Name Plate } \\
\text { Rating (KW) }\end{array}$ & PESF & \multicolumn{1}{c}{ PESF*KW } & $\begin{array}{l}\text { Annual } \\
\text { Rating }\end{array}$ & $\begin{array}{c}\text { Energy } \\
\text { (MWh) }\end{array}$ & $\begin{array}{c}\text { PESF Annual } \\
\text { Energy Rating (MWh) }\end{array}$ \\
28000.00 & 0.50 & 14000.00 & & 170000.00 & 85000 \\
Unit & Plant & Project & Dam & Latitude & 4131.00 \\
Type & Type & Status & Status & Longitude & 9032.00 \\
& ROR & & W & &
\end{tabular}

Factor

Wild/Scenic Protection Wild/Scenic Tributary or Upstream/Downstream wild/Scenic Location Cultural Value

Fish Presence Value

Geologic Value

Historic Value

Other Value

Recreation Value

Scenic Value
Exist Prob

Factor

Exist Prob

0.90

Wildlife value Threatened/Endangered Fish Threatened/Endangered Wildife Y

0.90

Federal Land Code 103

0.90

Federal Land Code 104

0.90

Federal Land Code 105

0.90

Federal Land Code 106

0.90 Federal Irand Code 107

Y 0.75 Federal Land Code 108

$Y \quad 0.75$ Federal Land Code 198
0.90

0.75

0.75

0.90

0.90

0.90

0.90

0.90

0.90

0.90 
DATE : $12 / 06 / 96$

PAGE NO: 10

FERC

Number

Plant Name

Stream

State

Name

07647

BROOKPORT (L\&D 52)

OHIO R

IL

County Name

MASSAC
River Basin

OHIO MAIN STREAM

Class Owner Name

R EROOKPORT ASSOC

Name Plate

PESF

Annual Energy

Rating (KW)

$0.50 \quad 17250.00$

Rating (MWh)

PESF Annual

34500.00

302000.00

Energy Rating

(MWh)

$\begin{array}{cccccc}\text { Unit } & \text { Plant } & \text { Project } & \text { Dam } & \text { Latitude } & 3707.00 \\ \text { Type } & \text { Type } & \text { Status } & \text { Status } & \text { Longitude } & 8839.00 \\ & & & \text { XX } & \text { WO } & \end{array}$

Factor

Exist Prob

Factor

Wild/Scenic Protection

wild/Scenic Tributary or

Upstream/Downstream

Wild/Scenic Location

Cultural Value

Fish Presence Value

Geologic Value

Historic Value

Other value

Recreation Value

Scenic Value

Exist Prob

0.90

Wildlife Value

Threatened/Endangered Fish Threatened/Endangered Wildlife

0.90 Federal Land Code 103

0.90 Federal Land Code 104

0.90 Federal Land Code 105

Y 0.90 Federal Land Code 106

0.90 Federal Land Code 107

$Y \quad 0.75$ Federal Land Code 108

$Y \quad 0.75$ Federal Land Code 198

\section{0}

0.90

Exist Prob

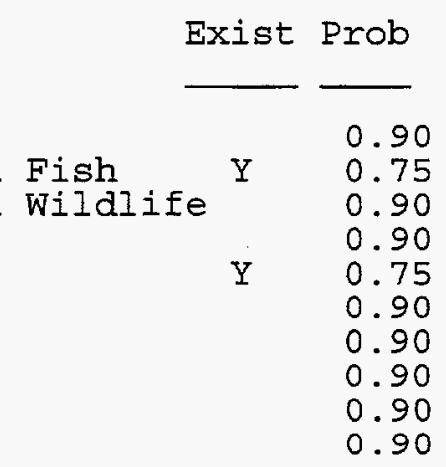


RES O URCE DA TA B A S E I I S T I N G

DATE : $12 / 06 / 96$

PAGE NO: 11

FERC

Number

Plant Name

Stream

State

08632 KANKAKEE

KANKAKEE RIVER

Name

II

\author{
County Name
}

River Basin

KANKAKEE

ILIINOIS RIVER BASIN

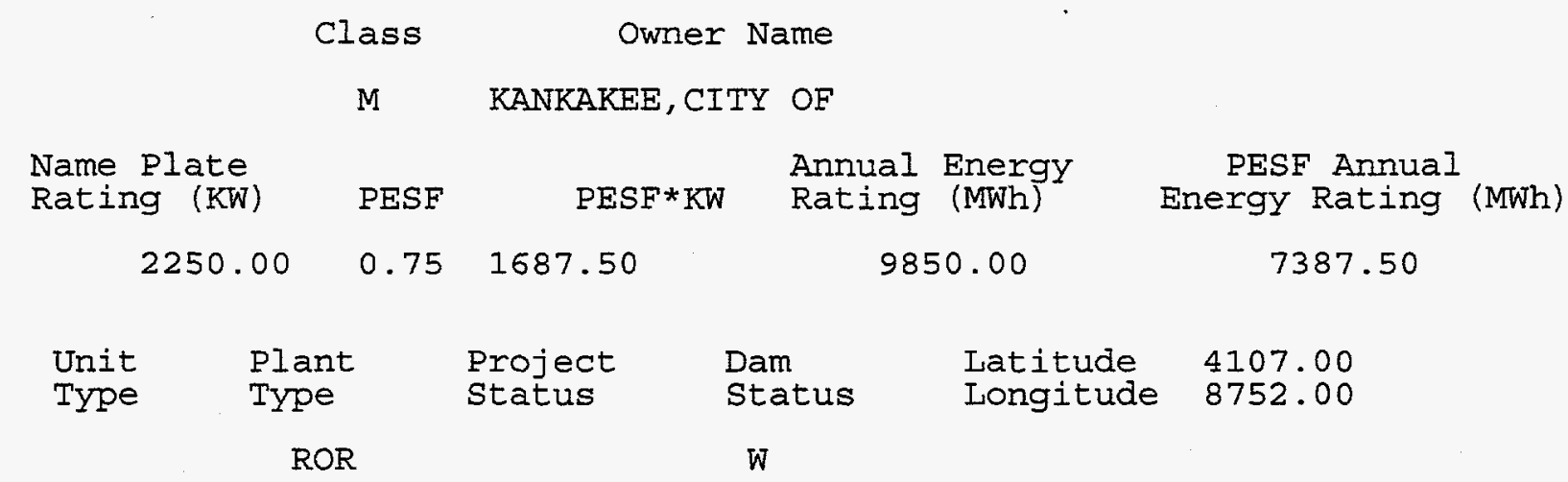

Factor

Wild/Scenic Protection

Wild/Scenic Tributary or Upstream/Downstream wild/Scenic Location Cultural Value Fish Presence Value

Geologic Value

Historic Value

Other Value

Recreation Value

Scenic Value
Exist Prob

Factor

0.90 Wildlife Value Threatened/Endangered Fish Threatened/Endangered Wildlife

0.90 Federal Land Code 103

0.90 Federal Land Code 104

0.90 Federal Land Code 105

0.90 Federal Land Code 106

0.90 Federal Iand Code 107

0.90 Federal Land Code 108

0.90 Federal Land Code 198

0.90
Exist Prob

\begin{tabular}{|c|c|c|}
\hline & xist & Prob \\
\hline $\begin{array}{l}\text { Fish } \\
\text { Wildlife }\end{array}$ & $Y$ & $\begin{array}{l}0.90 \\
0.75 \\
0.90 \\
0.90 \\
0.90 \\
0.90 \\
0.90 \\
0.90 \\
0.90 \\
0.90\end{array}$ \\
\hline
\end{tabular}

0.75

.90

0.90

0.90

. 

R E SOURCE
$D A T A B A S E$
I I S T N G

DATE : $12 / 06 / 96$

PAGE NO: 12

FERC

Number

08984
Plant Name

MISSISSIPPI L\&D 22

County Name

PIKE

\section{Stream}

MISSISSIPPI R

River Basin

SALT RIVER BASIN
State

Name

II

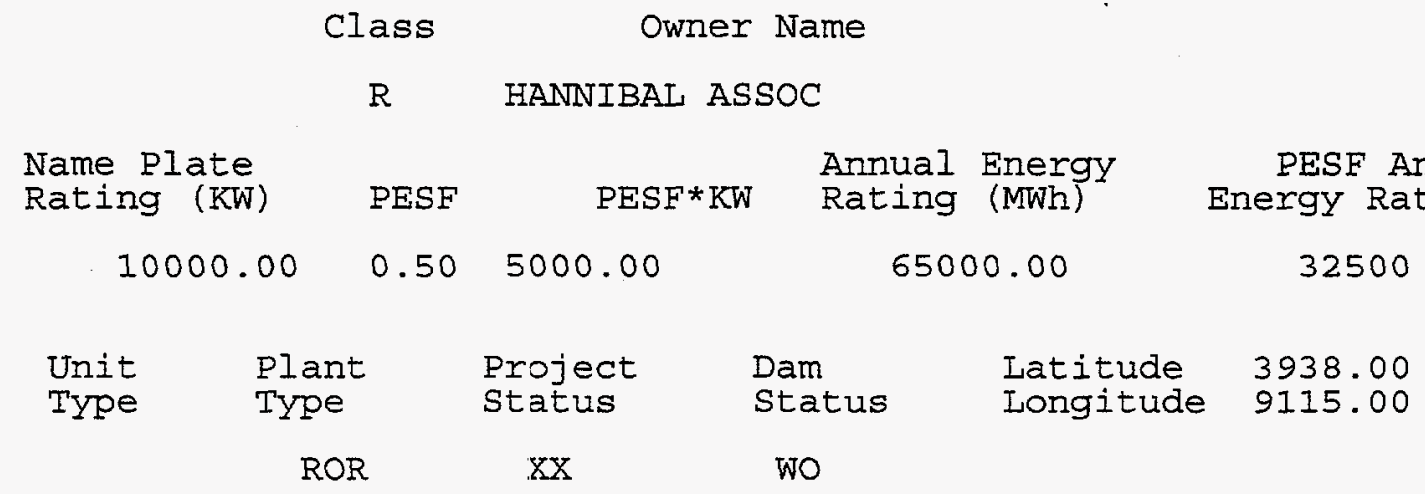

Factor

Exist Prob

Factor

Exist Prob

Wild/Scenic Protection

Wild/Scenic Tributary or Upstream/Downstream wild/Scenic Location Cultural Value

Fish Presence Value

Geologic Value

Historic Value

Other Value

Recreation Value

-

0.90

Wildife Value

Threatened/Endangered Fish

0.90

Threatened/Endangered Wildlife $Y$

0.90

Federal Land Code 103

0.90 Federal Land Code 104

0.90 Federal Land Code 105

0.90 Federal Land Code 106

0.90 Federal Land Code 107

Y $\quad 0.75$ Federal Land Code 108

Scenic Value

0.75

Federal Land Code 198

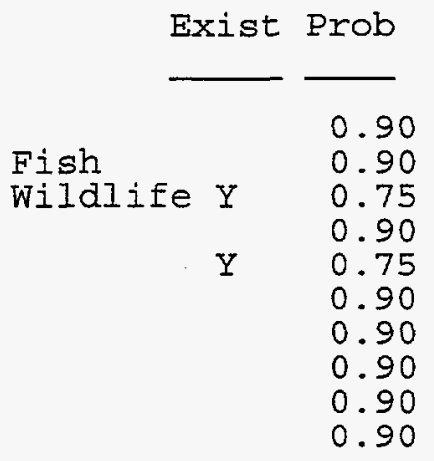


$R E S O U R C E \quad D A T A B A S E$ I I S T I N G

DATE : $12 / 06 / 96$

PAGE NO: 13

FERC

Number

Plant Name

Stream

State

09051

REND IAKEE DAM

BIG MUDDY $\mathrm{R}$

Name

County Name

River Basin

FRANKIIN

MINOR RIVER BASINS

Class Owner Name

$\mathrm{R}$ BENTON ASSOC

$\begin{array}{lll}\text { Name Plate } & & \text { Annual Energy } \\ \text { Rating (KW) PESF } & \text { PESF*KW Rating (MWh) }\end{array}$

PESF Annual
Energy Rating (MWh)

$$
1600.00 \quad 0.50 \quad 800.00
$$

5000.00

2500

$\begin{array}{lllll}\text { Unit } & \text { Plant } & \text { Project } & \text { Dam } & \text { Latitude } \\ \text { Type } & \text { Type } & \text { Status } & \text { Status } & \text { Longitude } 8858.00\end{array}$

ROR XX WO

Factor

Wild/Scenic Protection

Wild/Scenic Tributary or Upstream/Downstream

wild/Scenic Location

Cultural value

Fish Presence Value

Geologic Value

Historic Value

other Value

Recreation Value

Scenic Value
Exist Prob

\begin{tabular}{|c|c|}
\hline & 0.90 \\
\hline & $\begin{array}{l}0.90 \\
0.90 \\
0.90 \\
0.90 \\
0.90\end{array}$ \\
\hline $\begin{array}{l}Y \\
Y \\
Y\end{array}$ & $\begin{array}{l}0.75 \\
0.75 \\
0.90\end{array}$ \\
\hline
\end{tabular}

Factor

Exist Prob

Wildlife Value Threatened/Endangered Fish Threatened/Endangered Wildlife Federal Land Code 103 Federal Land Code 104 Federal Land Code 105 Federal Land Code 106 Federal Land Code 107 Federal Land Code 108 Federal Iand Code 198
II 

R E S
$U$ R $C E$
D A T A B A S E
I I $S I N G$

DATE : $12 / 06 / 96$

PAGE NO: 14

FERC

Number

Plant Name

stream

State

09084 MISSISSIPPI I\&D 20

MISSISSIPPI $R$

Name

09084

County Name

River Basin

ADAMS

SALT RIVER BASIN

IL

Class Owner Name

$\mathrm{R}$ CANTON ASSOC

Name Plate
Rating (KW)

PESF

Annual Energy

Rating ( $\mathrm{KW}$ )

PESF*KW

Rating (MWh)

30000.00

$0.50 \quad 15000.00$

79000.00

PESF Annual
Energy Rating (MWh)

Unit Plant

Type

Type

ROR

Factor

Exist Prob

0.90

Wild/Scenic Protection

Wild/Scenic Tributary or

Upstream/Downstream

wild/Scenic Location

Cultural Value

Fish Presence Value

Geologic Value

Historic Value

other Value

Recreation Value

Scenic Value
Project

status

$\mathrm{XX}$
Dam
Status

Latitude Longitude

4309.00

WO
39500 

R E S O U R C E
$\mathrm{D} A \mathrm{~T} A \mathrm{~A} A \mathrm{~S} \mathrm{E}$
I I S T I N G

DATE : $12 / 06 / 96$

PAGE NO: 15

FERC

Number

Plant Name

stream

State

09256 KASKASKIA L\&D

KASKASKIA R

Name

County Name

River Basin

RANDOLPH

KASKASKIA RIVER BASIN

$\begin{array}{cc}\text { Class } & \text { Owner Name } \\ \text { R } & \text { RANDOLPH HYDRO ASSOC }\end{array}$

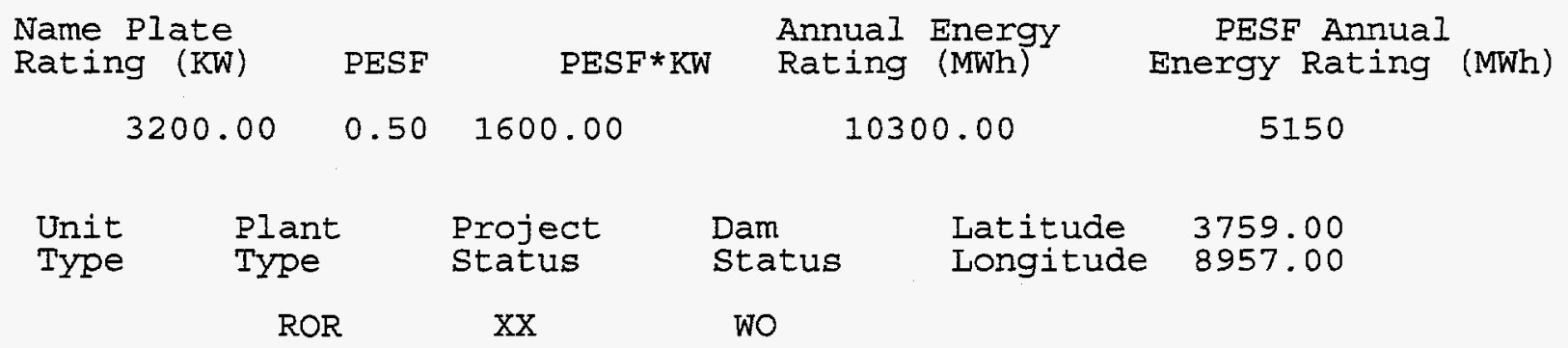

Factor

Wild/Scenic Protection wild/Scenic Tributary or Upstream/Downstream Wild/Scenic Location Cultural value

Fish Presence Value

Geologic Value

Historic Value

other Value

Recreation value

Scenic value
Exist Prob

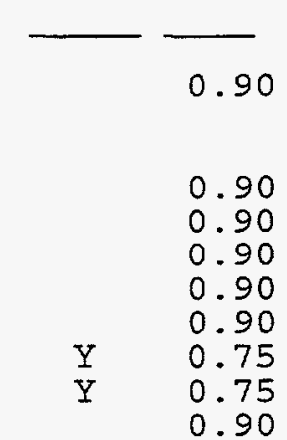

Factor

Exist Prob

Wildlife Value Threatened/Endangered Fish $Y \quad 0.75$ Threatened/Endangered Wildife $Y \quad 0.75$ Federal Land Code 103 Federal Land Code 104 Federal Land Code 105 Federal Land Code 106 Federal Land Code 107 Federal Land Code 108 Federal Land Code 198
II 

$R E S O U R C E$
D A T A B A $S E$
I I S T I N G

DATE : $12 / 06 / 96$

PAGE NO: 16

FERC

Number

Plant Name

stream

State

09828

WILMINGTON

KANKAKEE R

Name

County Name

River Basin

WILI

ILIINOIS RIVER BASIN

Class Owner Name

$R$ COLLINS, THOMAS $J$

Name Plate
Rating $(\mathrm{KW})$

PESF

Annual Energy

PESF*KW

Rating (MWh)

PESF Annual

600.00

$0.50 \quad 300.00$

3800.00

Energy Rating (MWh)

$\begin{array}{cc}\text { Unit } & \text { Plant } \\ \text { Type } & \text { Type } \\ & \text { ROR }\end{array}$

Project

Dam

Status

Iatitude Longitude

ROR

XX

WO

IL

Factor

Wild/Scenic Protection

wild/scenic Tributary or

Upstream/Downstream

Wild/Scenic Location

Cultural Value

Fish Presence Value

Geologic Value

Historic Value

Other Value

Recreation Value

Scenic Value
Exist Prob

Factor

Exist Prob

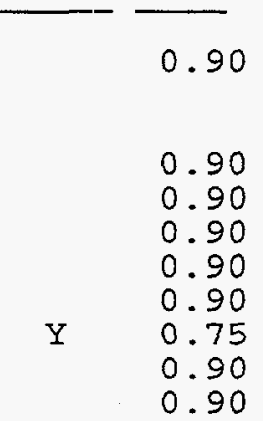

Wildlife Value

Threatened/Endangered Fish Threatened/Endangered Wildlife

Federal Land Code 103

Federal Land Code 104

Federal Land Code 105

Federal Land Code 106

Federal Land Code 107

Federal Land Code 108

Federal Land Code 198
4118.00

1900

8809.00 

$R E S O U R$
C E
D T A B A S E
L I S T I N G

DATE : $12 / 06 / 96$

PAGE NO: 17

FERC

Number

Plant Name

stream

State

Name

09829 FORDAM

ROCK R

IL

County Name

WINNEBAGO

River Basin

ROCK RIVER BASIN

\section{Class Owner Name}

$R$ COLLINS, THOMAS J

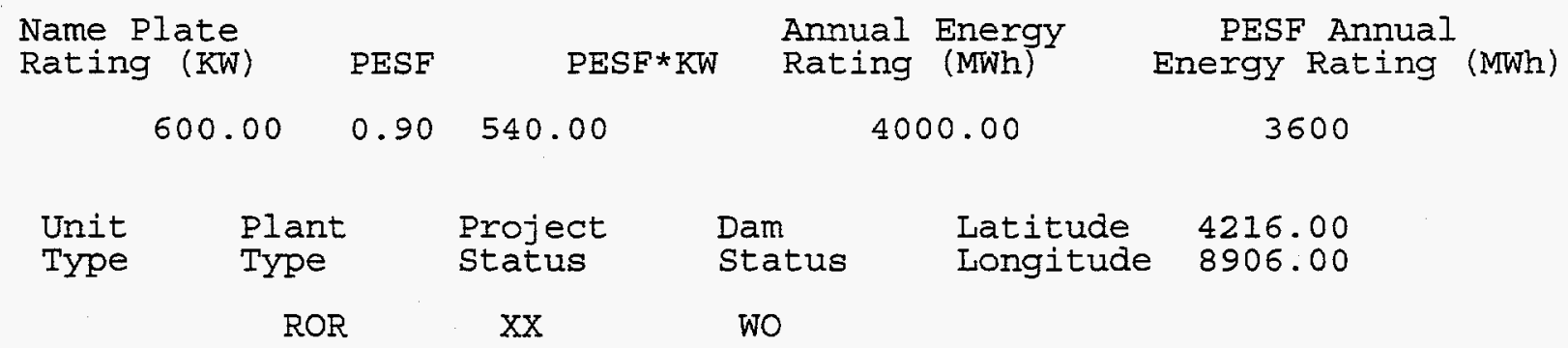

Factor

Wild/scenic Protection

Wild/Scenic Tributary or Upstream/Downstream wild/Scenic Location

Cultural Value

Fish Presence Value

Geologic Value

Historic Value

other Value

Recreation Value

Scenic Value
Exist Prob

0.90

Wildlife Value Threatened/Endangered Fish Threatened/Endangered Wildlife

0.90 Federal Land Code 103

0.90 Federal Land Code 104

0.90 Federal Land Code 105

0.90 Federal Land Code 106

0.90 Federal Land Code 107

0.90 Federal Irand Code 108

0.90 Federal Land Code 198
Exist Prob

0.90

0.90

0.90

0.90

0.90

0.90

0.90

0.90

0.90

0.90 
DATE : $12 / 06 / 96$

PAGE NO: 18

FERC

Number

10423

LAKE

Plant Name

County Name

COLES

Class

Owmer Name

M. CHARLESTON, CITY OF

Name Plate

Rating (KW)

PESF

$\mathrm{PESF}^{\star} \mathrm{KW}$

Annual Energy

700.00

$0.50 \quad 350.00$

2800.00

Dam Latitude

Status

Longitude

Status

IXX

WO

$\begin{array}{cccccc}\text { Unit } & \text { Plant } & \text { Project } & \text { Dam } & \text { Latitude } & 3927.00 \\ \text { Type } & \text { Type } & \text { Status } & \text { Status } & \text { Longitude } 8809.00 \\ & \text { ROR } & \text { IXX } & \text { WO } & \end{array}$

State

Name

II
Factor

Wild/Scenic Protection

Wild/Scenic Tributary or Upstream/Downstream wild/Scenic Location Cultural Value

Fish Presence Value

Geologic Value

Historic Value

other value

Recreation Value

Scenic Value
Exist: Prob

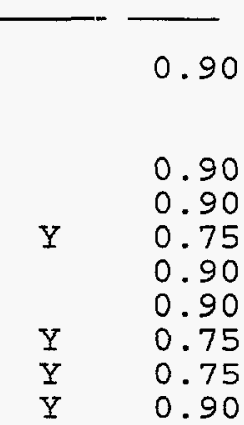

Factor

Exist Prob

Wildife value Threatened/Endangered Fish Federal Land Code 103 Federal Land Code 104 Federal Land Code 105 Federal Land Code 106 Federal Land Code 107

Federal Land Code 108

Federal Land Code 198
PESF Annual Energy Rating (MWh)

1400 
RESOURCE D A T A B A S E L I S T I N G

DATE : $12 / 06 / 96$

PAGE NO: 19

FERC

Number

Plant Name

Stream

State

11214 CARLYLE

KASKASKIA $\mathrm{R}$

Name

County Name

River Basin

CLINTON

KASKASKIA RIVER BASIN

Class Owner Name

C SOUTHWESTERN ELEC COOP INC

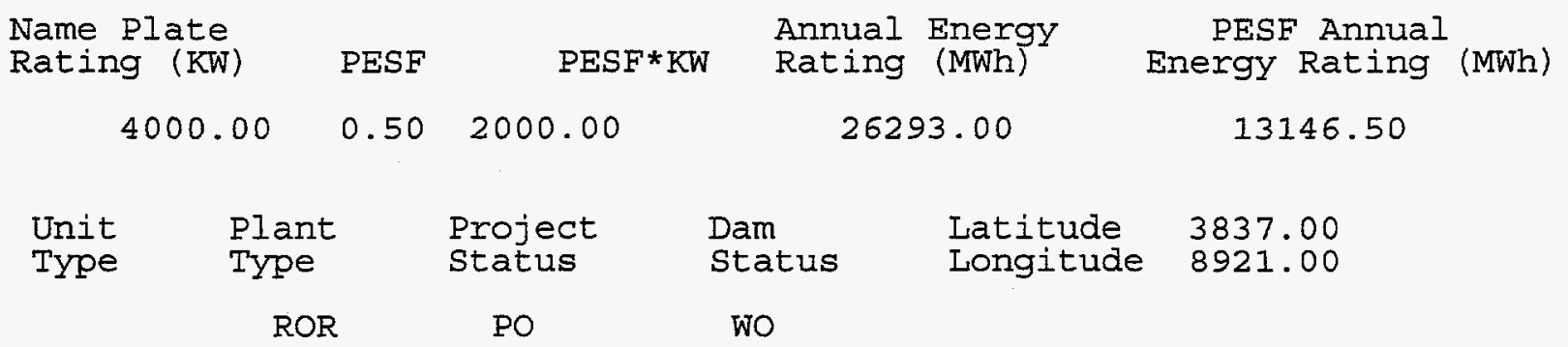

Factor

Wild/Scenic Protection

Wild/scenic Tributary or Upstream/Downstream wild/Scenic Location Cultural Value

Fish Presence Value

Geologic Value

Historic Value

other value

Recreation Value

Scenic Value
Exist Prob

\subsection{0}

0.90

0.90

0.90

0.90

0.90

$\begin{array}{ll}Y & 0.75 \\ Y & 0.75\end{array}$

$\begin{array}{ll}Y & 0.75 \\ Y & 0.90\end{array}$
Factor

Exist Prob

Wildlife Value Threatened/Endangered Fish Threatened/Endangered Wildlife Federal Land Code 103 Federal Land Code 104 Federal Land Code 105 Federal Land Code 106 Federal Land Code 107 Federal Land Code 108 Federal Iand Code 198

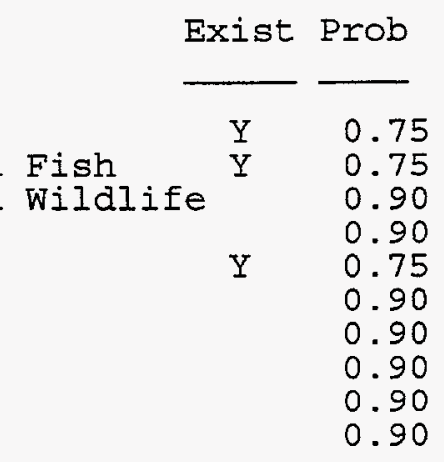

IL 

$R E S O U R C E$
D A T A B A S E
I I S T I N G

DATE : $12 / 06 / 96$

PAGE NO: 20

FERC

Number

Plant Name

Stream

State

11223 L\&D 24 HYDROELECTRIC

MISSISSIPPI RIVER

Name

IL

County Name

CALHOUN
River Basin

MISSISSIPPI RIVER BASIN
Class Owner Name

P L\&D 24 HYDRO ASSOCIATES

Name Plate Rating (KW)

PESF

Annual Energy

$\mathrm{PESF} * \mathrm{KW}$

Rating

(MWh)

50000.00

0.50

25000.00

245000.00

Project

Status

ROR

Exist Prob

Factor

Latitude Irongitude

3923.00

Status

WO

\begin{tabular}{|c|c|c|c|c|c|}
\hline $\begin{array}{l}\text { Unit } \\
\text { Type }\end{array}$ & $\begin{array}{l}\text { Plant } \\
\text { Type }\end{array}$ & $\begin{array}{l}\text { Project } \\
\text { Status }\end{array}$ & $\begin{array}{l}\text { Dam } \\
\text { Status }\end{array}$ & $\begin{array}{l}\text { Latitude } \\
\text { Longitude }\end{array}$ & $\begin{array}{l}3923.00 \\
9054.00\end{array}$ \\
\hline & ROR & & wo & & \\
\hline
\end{tabular}

PESF Annual Energy Rating (MWh)

122500

\author{
Factor \\ Wild/Scenic Protection \\ Wild/Scenic Tributary or \\ Upstream/Downstream \\ wild/Scenic Location \\ Cultural Value \\ Fish Presence Value \\ Geologic Value \\ Historic Value \\ Other Value \\ Recreation Value \\ Scenic Value
}

Exist

0.90 Wildlife Value

Threatened/Endangered Fish

Exist Prob

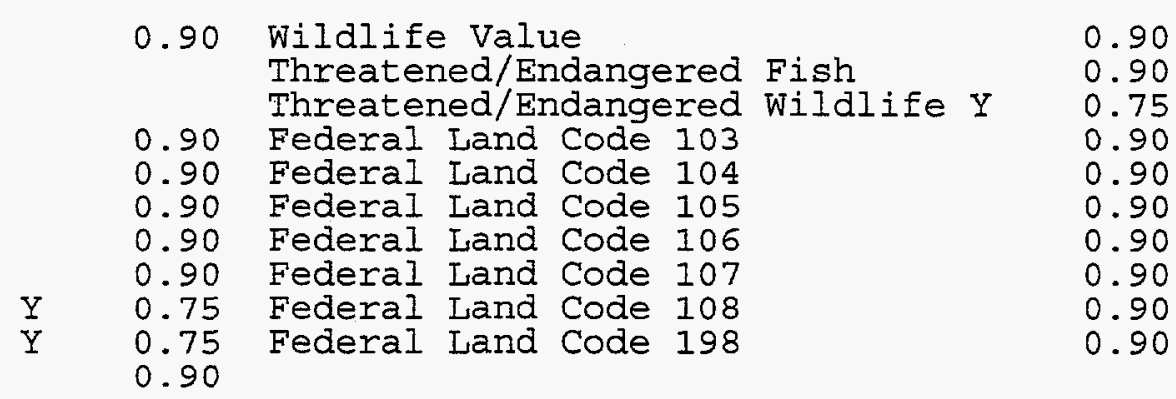


DATE: $12 / 06 / 96$

PAGE NO: 21

FERC

Number

Plant Name

Stream

State

11224 L\&D 25 HYDROELECTRIC

MISSISSIPPI RIVER

Name

11224 L\&D 25 HYDROELECTRIC
County Name
River Basin

CALHOUN

MISSISSIPPI RIVER BASIN

IL

$\begin{array}{cc}\text { Class } & \text { Owner Name } \\ \text { P } & \text { L\&D } 25 \text { HYDRO ASSOCIATES }\end{array}$

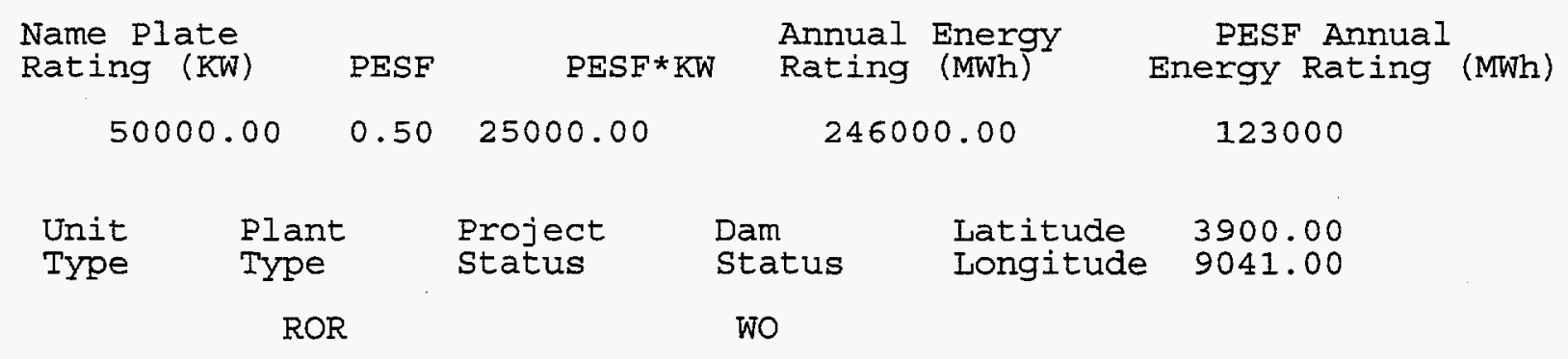

\section{Factor}

Exist Prob

Factor

Exist Prob

wild/Scenic Protection

wild/Scenic Tributary or Upstream/Downstream wild/Scenic Location Cultural Value

Fish Presence value

Geologic Value

Historic Value

other Value

Recreation Value

Scenic Value

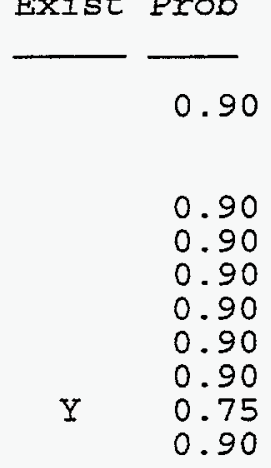

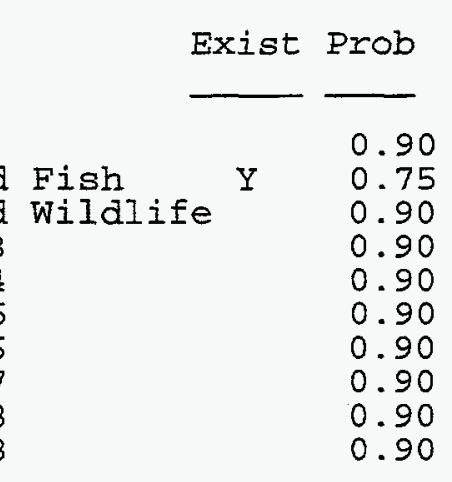

wildife value Threatened/Endangered Fish Threatened/Endangered Wildlife Federal Land Code 103 Federal Land Code 104 Federal Land Code 105 Federal Land Code 106 Federal Iand Code 107 Federal Land Code 108 Federal Land Code 198
0.90

0.75

0.90

0.90

0.90 

R E S O U R C E
D A T A B A S E
L I S T I N G

DATE : $12 / 06 / 96$

PAGE NO: 22

FERC

Number

11307
Plant Name

MARSEILLES HYDROELECTRIC

County Name

LASALLE
Stream

ILIINOIS RIVER

River Basin

ILIINOIS RIVER BASIN

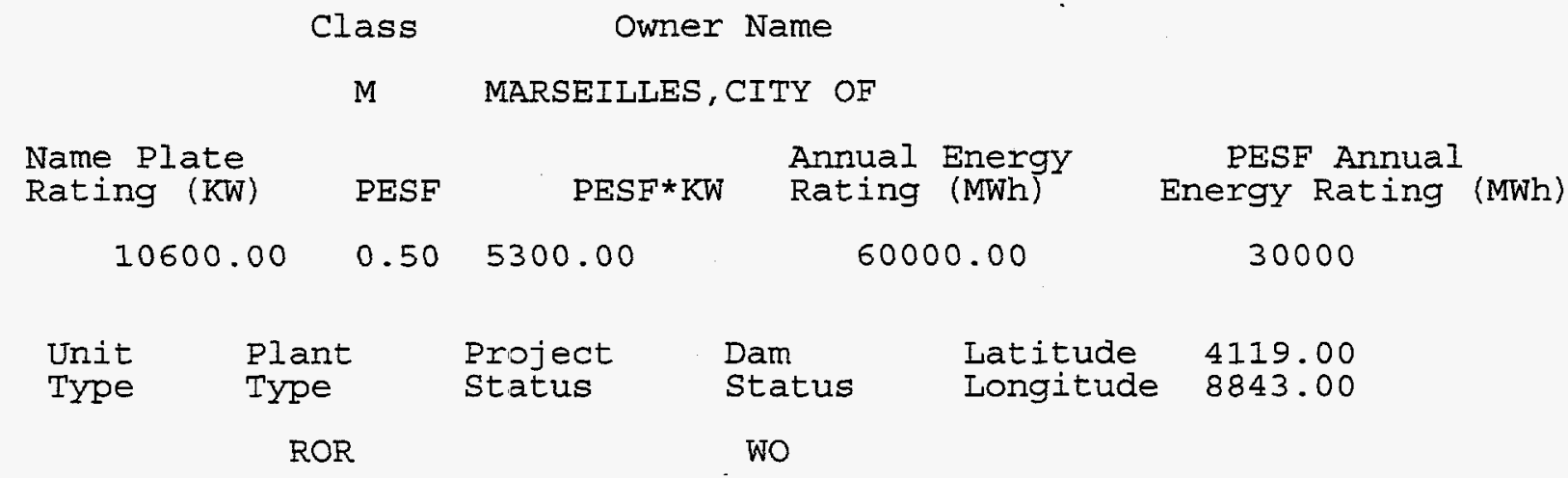

Factor

Wild/scenic Protection

Wild/Scenic Tributary or

Upstream/Downstream

Wild/Scenic Location

Cultural Value

Fish Presence Value

Geologic Value

Historic Value

Other Value

Recreation Value

Scenic Value
Exist Prob

Factor

0.90 Wildlife Value

Threatened/Endangered Fish Threatened/Endangered Wildlife

Exist Prob

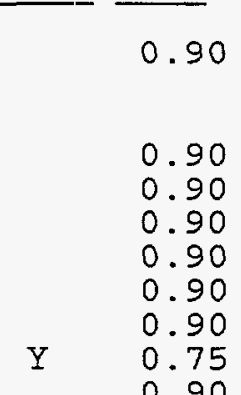

Federal Land Code 103

Federal Land Code 104

Federal Land Code 105

Federal Land Code 106

Federal Iand Code 107

Federal Land Code 108

0.75 Federal Iand Code 198

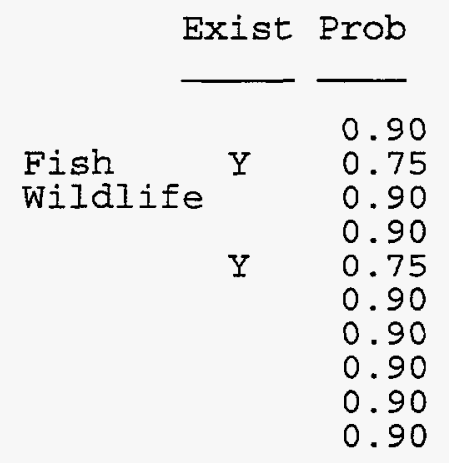

Name

II 

R E SOUR C E
D A T A B A S E
I I S I N G

DATE : $12 / 06 / 96$

PAGE NO: 23

FERC

Number

Plant Name

Stream

State

11360 OREGON

ROCK R

Name

\section{OREGON}

County Name

River Basin

OGLE

ROCK RIVER BASIN

$\begin{array}{cc}\text { Class } & \text { Owner Name } \\ \text { R } & \text { THOMAS J.COLLINS \& ANDREW R.BLYSTRA }\end{array}$

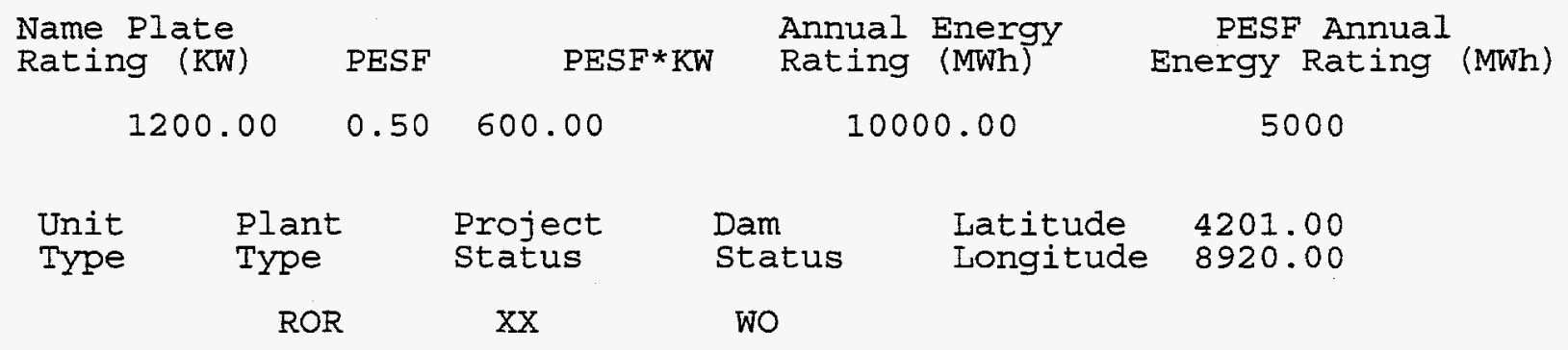

Factor

Exist Prob

$$
0.90
$$

Wild/Scenic Protection Wild/Scenic Tributary or Upstream/Downstream wild/scenic Location Cultural Value

Fish Presence Value

Geologic Value

Historic value

Other Value

Recreation Value

Scenic Value

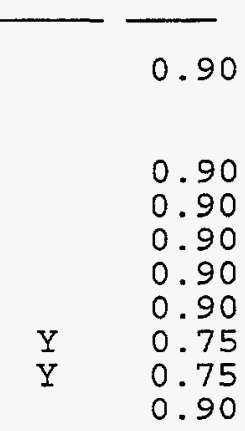

Wildlife Value Threatened/Endangered Fish Threatened/Endangered Wildlife

Exist Prob Federal Land Code 103 Federal Land Code 104 Federal Land Code 105 Federal Land Code 106 Federal Land Code 107 Federal Land Code 108 Federal Land Code 198

\begin{tabular}{ll} 
Exist & Prob \\
\cline { 2 - 2 } Fish & \\
\hline & 0.90 \\
Wildife & 0.90 \\
4 & 0.90 \\
6 & 0.90 \\
7 & 0.90 \\
8 & 0.90 \\
8 & 0.90 \\
& 0.90 \\
& 0.90 \\
& 0.90
\end{tabular}


DATE : $12 / 06 / 96$

PAGE NO: 24

FERC

Number

Plant Name

Stream

State

11380

GENEVA

FOX R

County Name

KANE
River Basin

IIIINOIS RIVER BASIN

\section{Class Owner Name}

$R$ SHO-DEEN, INCNG

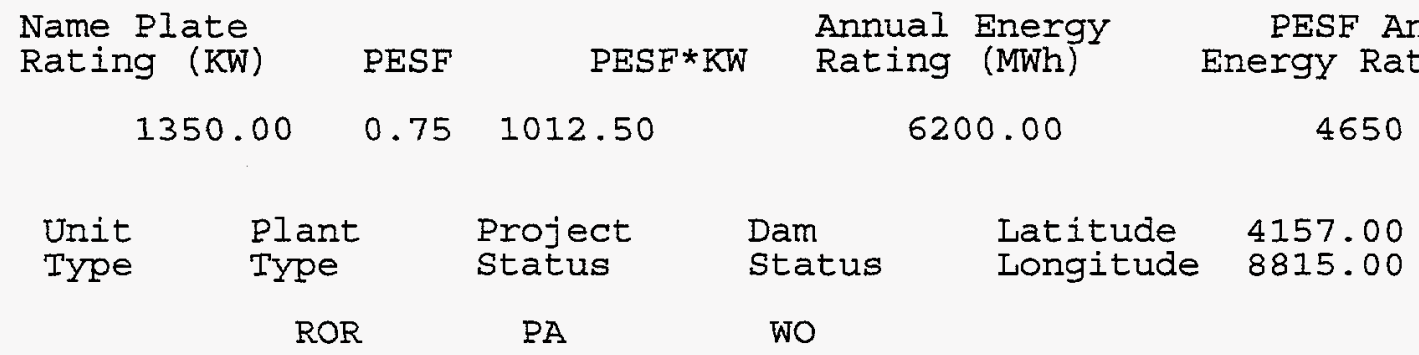

Factor

Wild/Scenic Protection wild/Scenic Tributary or Upstream/Downstream Wild/scenic Location Cultural Value

Fish Presence Value

Geologic Value

Historic Value

other Value

Recreation Value

Scenic Value
Exist Prob

Factor

0.90 Wildlife Value

Threatened/Endangered Fish

Threatened/Endangered Wildlife

Exist Prob

0.90 Federal Land Code 103

0.90 Federal Land Code 105

0.90 Federal Iand Code 106

0.90 Federal Land Code 107

0.90 Federal Land Code 108

$\mathrm{Y}$

0.75

0.90
IL

Name 

R E S O U R C E
D A T A B A S E
L I S T I N G

DATE : $12 / 06 / 96$

PAGE NO: 25

FERC

Number Plant Name

stream

State

11559 MARSEILIES L\&D

ILLINOIS $R$

Name

County Name

River Basin

IA SAILE

IILINOIS RIVER BASIN

Class Owner Name

M OGLESBY, TOWN OF

$\begin{array}{ccccc}\begin{array}{l}\text { Name Plate } \\ \text { Rating (KW) }\end{array} & \text { PESF } & \text { PESF*KW } & \begin{array}{l}\text { Annual Energy } \\ \text { Rating }\end{array} & \begin{array}{c}\text { PESF Annual } \\ \text { (MWh) }\end{array} \\ 6840.00 & 0.50 & 3420.00 & 48000.00 & 24000\end{array}$

Unit Plant Project Dam Latitude 4119.00

Type Type Status Status Longitude 8843.00

ROR PO WO

Factor

Factor

Exist Prob

Wild/Scenic Protection

wild/scenic Tributary or Upstream/Downstream wild/Scenic Location Cultural Value

Fish Presence Value

Geologic Value

Historic Value

Other Value

Recreation Value

Scenic Value

Exist Prob

0.90

0.90

0.90

0.90

0.90

0.90

$Y \quad 0.75$

0.90
Wildlife Value

Threatened/Endangered Fish Threatened/Endangered Wildlife Federal Land Code 103

Federal Land Code 104

Federal Land Code 105

Federal Land Code 106

Federal Land Code 107

Federal Land Code 108

Federal Land Code 198
IL 
R E S O U R E D A T A B A S E I I S T I N G

DATE : $12 / 06 / 96$

PAGE NO: 26

FERC

Number

Plant Name

Stream

State

11567 SHELBYVILLE

KASKASKIA R

Name

County Name

River Basin

SHELBY

KASKASKIA RIVER BASIN

Class Owner Name

M SHELBY ELECTRIC CORP

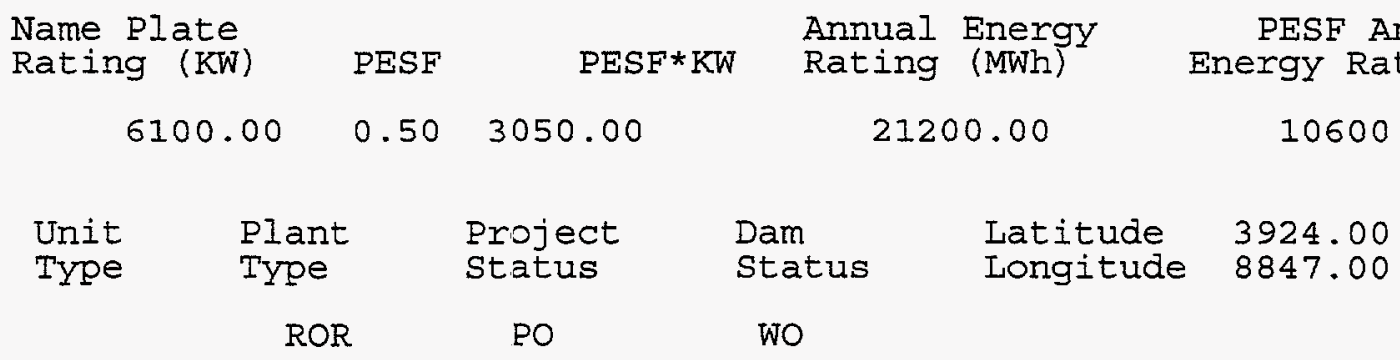

Factor

Exist Prob

Factor

Exist Prob

Wild/Scenic Protection

Wild/Scenic Tributary or Upstream/Downstream wild/Scenic Location Cultural Value

Fish Presence Value

Geologic Value

Historic Value

other Value

Recreation Value

Scenic Value

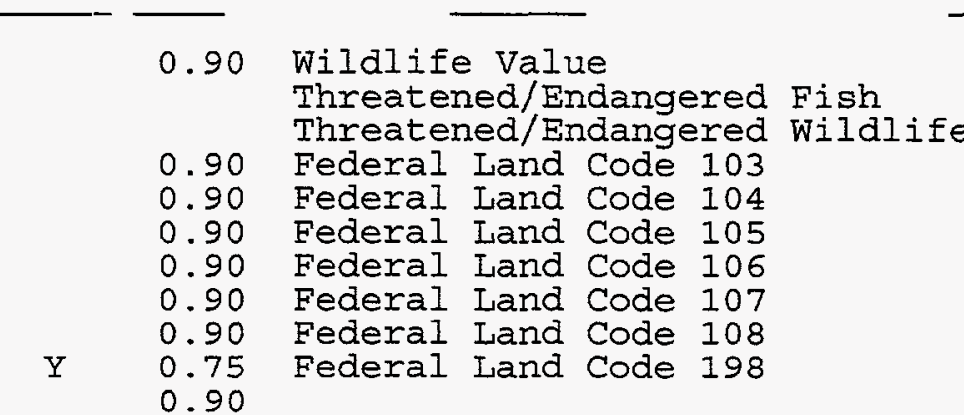

II

0.90

$\quad \begin{aligned} & 0.90 \\ & 0.90 \\ & 0.90 \\ & 0.75 \\ & 0.90 \\ & 0.90 \\ & 0.90 \\ & 0.90 \\ & 0.90\end{aligned}$


RESOURCE DA TABASE I I S T ING

DATE : $12 / 06 / 96$

PAGE NO: 27

FERC

Number

Plant Name

Stream

State

Name

IL001 IINCOLN

EMBARRASS R

II

County Name

River Basin

CUMBERL_AND

WABASH RIVER BASIN

Class Owner Name

NHPS/COE

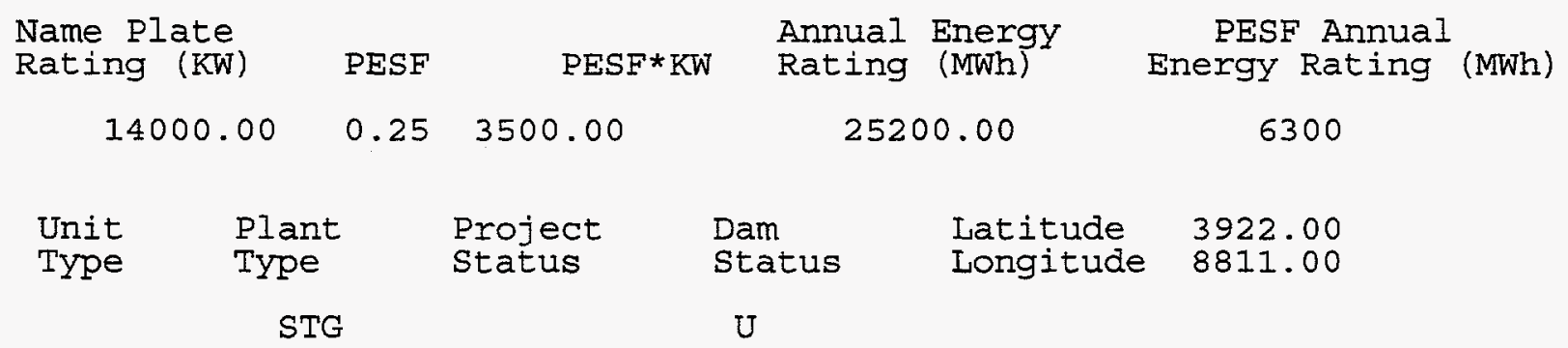

Factor

Exist Prob

Factor

Wild/Scenic Protection

wild/scenic Tributary or Upstream/Downstream wild/scenic Location Cultural value

Fish Presence value

Geologic Value

Historic Value

Other Value

Recreation Value

Scenic Value

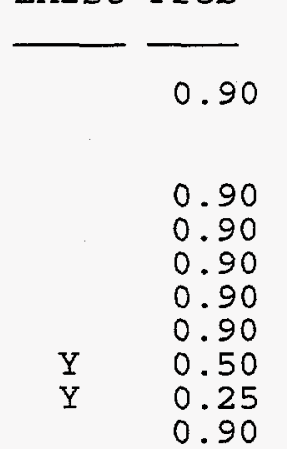

Exist Prob

$\begin{array}{lll} & \text { Exist } & \text { Prob } \\ & & \\ \text { Fish } & & 0.90 \\ \text { Wildife } & 0.50 \\ 3 & 0.90 \\ 7 & & 0.90 \\ 6 & & 0.90 \\ 7 & & 0.90 \\ 8 & & 0.90 \\ 8 & & 0.90 \\ & & 0.90 \\ & & 0.90\end{array}$

.90

.90

0.90

0.90 wildlife Value Threatened/Endangered Threatened/Endangered
Federal Land Code 103 Federal Land Code 104 Federal Land Code 105 Federal Land Code 106 Federal Land Code 107

Federal Land Code 108

Federal Land Code 198 
FERC

Number

Plant Name

ILOO2

DANVILLE

County Name

VERMIIION

Class

Owner Name

Name Plate

Rating (KW)

PESF

$\mathrm{PESF} * \mathrm{KW}$

Annual Energy

10000.00

$0.50 \quad 5000.00$

25000.00

Dam

Unit

Plant

Type

Project

status

STG

Exist Prob

Factor

Latitude Longitude

WO
River Basin

WABASH RIVER BASIN
State Name

IL

\author{
Factor \\ Wild/Scenic Protection \\ wild/Scenic Tributary or \\ Upstream/Downstream \\ wild/Scenic Location \\ Cultural value \\ Fish Presence Value \\ Geologic Value \\ Historic VaIue \\ other Value \\ Recreation Value \\ Scenic Value
}

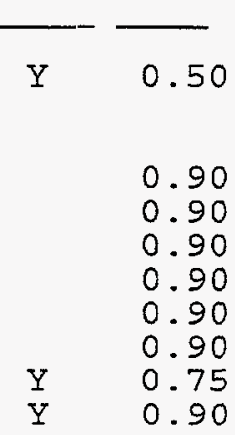

PESF Annual Energy Rating (MWh)

12500

4009.00

8739.00
Wildlife Value

Threatened/Endangered Fish Threatened/Endangered Wildlife

Federal Land Code 103

Federal Land Code 104

Federal Iand Code 105

Federal Land Code 106

Federal Land Code 107

Federal Land Code 108

Federal Iand Code 198
Exist Prob

0.90

0.75

0.90

0.90

0.90

0.90

0.90

0.90

0.90

0.90 

R E S O U R C E
D A T A B A S E
L I S T I N G

DATE : $12 / 06 / 96$

PAGE NO: 29

FERC

Number

Plant Name

Stream

State

IT 003

DAYTON

FOX R

Name

12003

County Name

River Basin

IAA SALLE

ILLINOIS RIVER BASIN

Class Owner Name

$P$ HYDRO-OP ONE ASSOCIATES

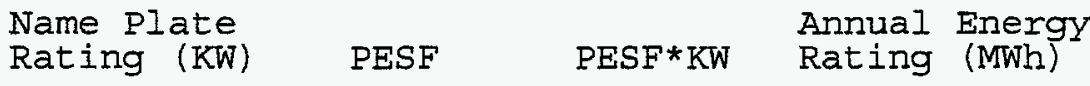

PESF Annual

630.00

$0.50 \quad 315.00$

7240.00

Energy Rating (MWh)

$\begin{array}{lllll}\text { Unit } & \text { Plant } & \text { Project } & \text { Dam } & \text { Latitude } 4123.00 \\ \text { Type } & \text { Type } & \text { Status } & \text { Status } & \text { Longitude } 8848.00\end{array}$

ROR

W

\section{Factor}

Wild/scenic Protection

Wild/scenic Tributary or Upstream/Downstream

wild/Scenic Location

Cultural Value

Fish Presence Value

Geologic Value

Historic Value

other Value

Recreation Value

Scenic Value
Exist Prob

0.90

0.90

0.90

0.90

$\mathrm{Y} \quad 0.90$

0.90

$\begin{array}{ll}Y & 0.75 \\ Y & 0.75\end{array}$

$\begin{array}{ll}Y & 0.75 \\ Y & 0.90\end{array}$
Factor

Exist Prob

Wildiffe Value

Threatened/Endangered Fish

Threatened/Endangered Fish

Wildife $\quad 0.90$

Federal Iand Code 103

Federal Iuand Code 104

Federal Land Code 105

Federal Land Code 106

Federal Land Code 107

Federal Land Code 108

Federal Land Code 198
0.90

0.90

0.90

0.90

0.90

0.90

0.90 

$R E S O U R C E$
D A T A B A S E
L I S T I N G

DATE : $12 / 06 / 96$

PAGE NO: 30

FERC

Number

Plant Name

Stream

State

ILOO4

WEDRON

FOX $R$

Name

County Name

River Basin

IA SALLE

ILIINOIS RIVER BASIN

Class Owner Name

Name Plate

PESF

Annual Energy

PESF Annual

Rating ( $\mathrm{KW})$

$0.50 \quad 4000.00$

48000.00

Energy Rating (MWh)

8000.00

Unit Plant Project

Type

Type

Status

Dam
Status
wo

Latitude

24000

STG

Factor

Wild/Scenic Protection

Wild/Scenic Tributary or

Upstream/Downstream

wild/Scenic Location

Cultural value

Fish Presence Value

Geologic Value

Historic Value

other Value

Recreation Value

Scenic Value
Exist Prob

$$
0.90
$$

Wildlife Value

Threatened/Endangered Fish

Threatened/Endangered Wildlife

0.90

Federal Iand Code 103

0.90 Federal Land Code 104

0.90 Federal Land Code 105

$Y \quad 0.90$

Federal Iand code 106

Federal Land Code 107

0.90

$Y \quad 0.75$

$Y \quad 0.75$

Federal Land Code 108

Federal Land Code 198
IL 
$R E S O U R C E$ D A T A B A S I I S T I N G

DATE : $12 / 06 / 96$

PAGE NO: 31

FERC

Number

Plant Name

Stream

State

IL005 MARSEILIES

ILIINOIS $R$

Name

County Name

River Basin

IA SALLE

ILLINOIS RIVER BASIN

Class Owner Name

$P$ ILLINOIS POWER CO

\begin{tabular}{|c|c|c|c|c|}
\hline $\begin{array}{l}\text { Name Plate } \\
\text { Rating (KW) }\end{array}$ & PESF & $\mathrm{PESF} * \mathrm{KW}$ & $\begin{array}{l}\text { Annual Energy } \\
\text { Rating (MWh) }\end{array}$ & $\begin{array}{l}\text { PESF Annual } \\
\text { Energy Rating (MWh) }\end{array}$ \\
\hline 2024.00 & 0.50 & 1012.00 & 16000.00 & 8000 \\
\hline
\end{tabular}

$\begin{array}{lllll}\text { Unit } & \text { Plant } & \text { Project } & \text { Dam } & \text { Latitude } 4119.00 \\ \text { Type } & \text { Type } & \text { Status } & \text { Status } & \text { Longitude } 8843.00\end{array}$

ROR WO

Factor

Exist Prob

Factor

Exist Prob

Wild/Scenic Protection

Wild/Scenic Tributary or Upstream/Downstream wild/Scenic Location

Cultural value

Fish Presence Value

Geologic Value

Historic Value

other Value

Recreation Value

Scenic Value

\begin{tabular}{|c|c|}
\hline & 0.90 \\
\hline$Y$ & $\begin{array}{l}0.90 \\
0.90 \\
0.90 \\
0.90 \\
0.90 \\
0.90 \\
0.75\end{array}$ \\
\hline$Y$ & 0.90 \\
\hline
\end{tabular}

IL 
DATE : $12 / 06 / 96$

PAGE NO: 32

FERC

Number

Plant Name

Stream

State

ILO06 MARSEILIES

ILLINOIS $\mathrm{R}$

County Name

IA SAILE
River Basin

ILLINOIS RIVER BASIN
Class

Owner Name

F

CORPS

OF ENGINEERS

Name Plate Rating (KW)

11000.00
Annual Energy Rating (MWh)

60000.00

Latitude Longitude

Project

Type

$$
\begin{aligned}
& \text { Plant } \\
& \text { Type }
\end{aligned}
$$

DIV

Factor

Wild/Scenic Protection

Wild/Scenic Tributary or

Upstream/Downstream

wild/Scenic Location

Cultural value

Fish Presence Value

Geologic Value

Historic Value

other Value

Recreation Value

Scenic Value
Exist Prob

0.90

0.90

0.90

0.90

0.90

0.90

0.90

$Y$
Dam

Status

WO
PESF Annual Energy Rating (MWh)

30000
IL

Name 

RESOURCE
D A $T$ A B A S E
I I $\mathrm{T} I \mathrm{NG}$

DATE : $12 / 06 / 96$

PAGE NO: 33

FERC

Number

Plant Name

Stream

State

IL007 OLD STATE

KANKAKEE R

Name

IL007 OLD STATE

County Name

River Basin

WILL

ILLINOIS RIVER BASIN

Class Owner Name

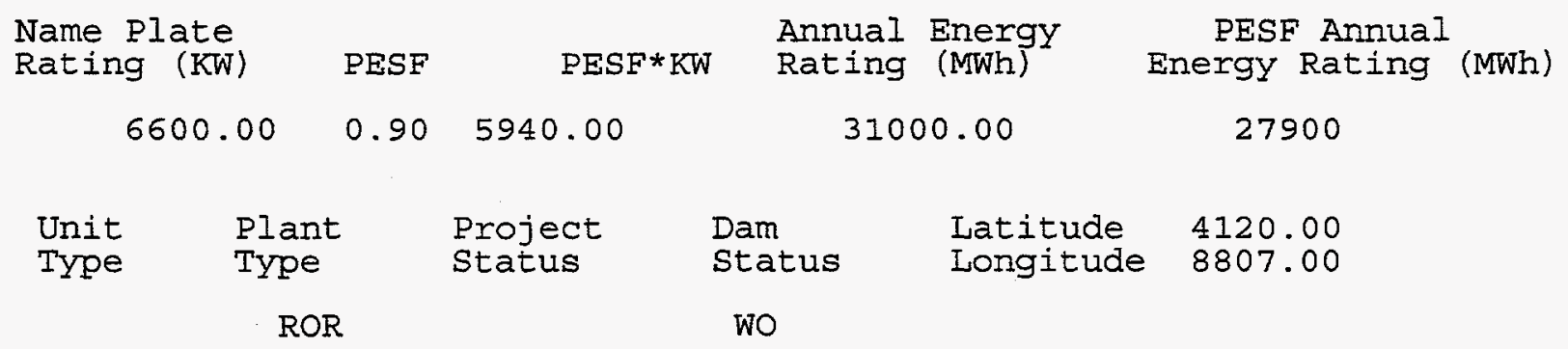

Factor

Wild/Scenic Protection Wild/Scenic Tributary or Upstream/Downstream wild/Scenic Location Cultural value Fish Presence Value Geologic Value Historic Value Other Value Recreation value Scenic Value

\section{Exist Prob}

Factor

Exist Prob

0.90

Wildlife Value Threatened/Endangered Fish Threatened/Endangered Wildlife

0.90 Federal Land Code 103

0.90

Federal Land Code 104

0.90

Federal Land Code 105

0.90$$
\text { Federal Land Code } 106
$$

0.90 Federal Land Code 107

0.90 Federal Land Code 108

0.90

Federal Iand Code 198
I工 
RES O URCE D A T A B A S E I I S T I $G$

DATE : $12 / 06 / 96$

PAGE NO: 34

FERC

Number

Plant Name

Stream

State

ILOO8 CUSTER PARK

KANKAKEE R

Name

county Name

WIIL

River Basin

ILIINOIS RIVER BASIN

Class Owner Name

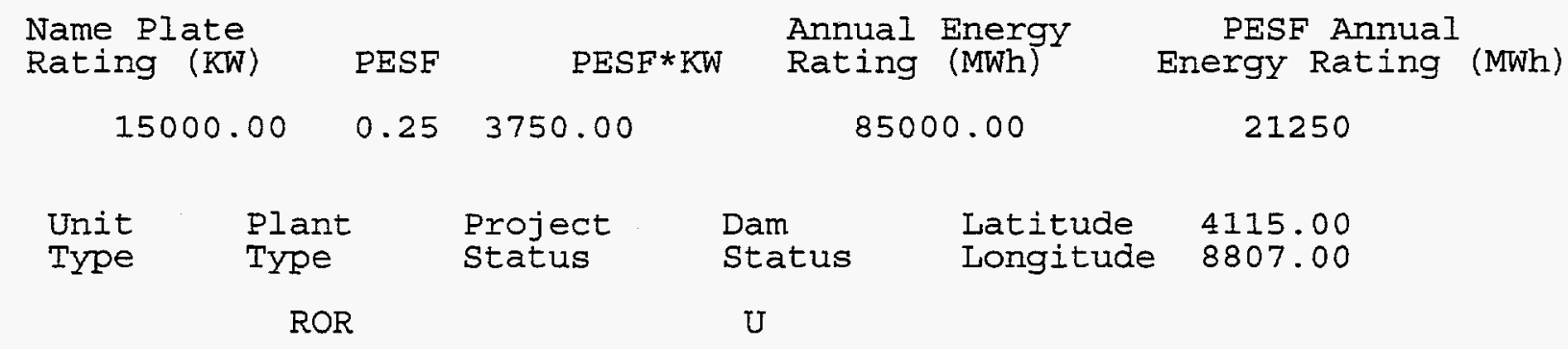

Factor

wild/Scenic Protection

wild/scenic Tributary or

Upstream/Downstream

wild/Scenic Location

Cultural Value

Fish Presence Value

Geologic Value

Historic Value

Other Value

Recreation Value

Scenic Value
Exist Prob

Factor

0.90 Wildlife Value

Threatened/Endangered Fish Threatened/Endangered Wildlife

0.90

0.90

0.90

0.90

0.90

$Y \quad 0.50$

0.90

0.90
Federal Land Code 103

Federal Land Code 104

Federal Land Code 105

Federal Land Code 106

Federal Land Code 107

Federal Land Code 108

Federal Land Code 198
IL
Exist Prob

0.90

0.50

0.90

0.90

0.90

0.90

0.90

0.90

0.90

0.90 
DATE : $12 / 06 / 96$

PAGE NO: 35

FERC

Number Plant Name

Stream

State

Name

IL009 BARSTOW

ROCK R

IL

County Name

River Basin

ROCK ISIAND

ROCK RIVER BASIN

Class Owner Name

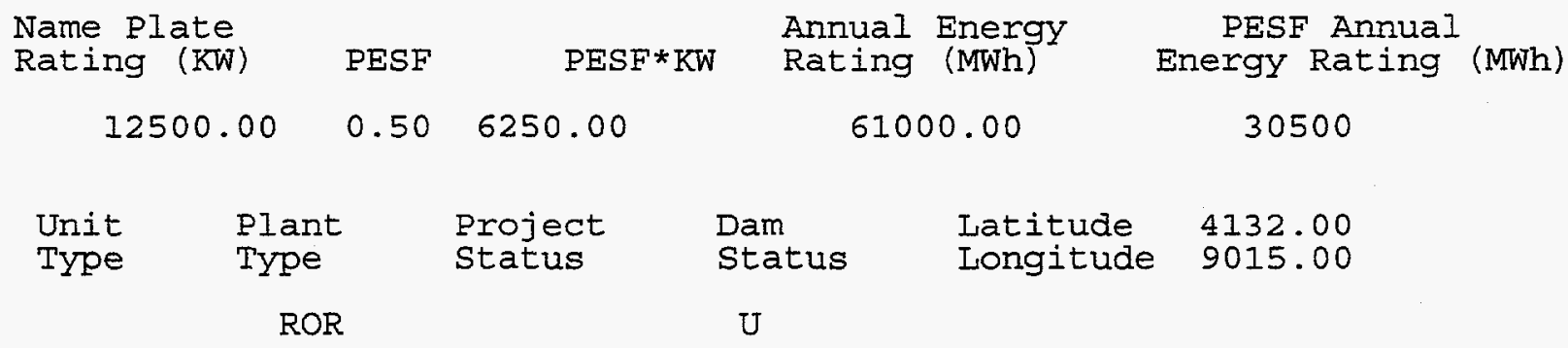

Factor

Exist Prob

Factor

Exist Prob

Wild/Scenic Protection

Wild/Scenic Tributary or

Upstream/Downstream

wild/Scenic Location

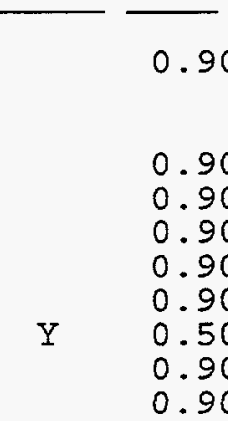

Wildlife Value

Threatened/Endangered Fish

Threatened/Endangered Wildlife

Federal Land Code 103

0.90 Federal Land Code 104

0.90 Federal Land Code 105

0.90 Federal Land Code 106

Fish Presence Value

Geologic Value

Historic Value

Other Value

Recreation Value

Scenic Value
Federal Iand Code 107

Federal Land Code 108

Federal Land Code 198
0.90

0.90

0.90

0.90

0.90

0.90

0.90

0.90

0.90

0.90 

R E SOU R C E
$D A T A B A S E$
I I S T I N G

DATE : $12 / 06 / 96$

PAGE NO: 36

FERC

Number

Plant Name

Stream

State

IIU010 DIXON

ROCK R

County Name

River Basin

LEE

ROCK RIVER BASIN

Class Owner Name

P STS HYDROPOWER LTD.

Name Plate

Rating ( $\mathrm{KW}$ )

PESF

Annual Energy

PESF Annual

4735.00

$0.50 \quad 2367.50$

$\begin{array}{lllll}\text { Unit } & \text { Plant } & \text { Project } & \text { Dam } & \text { Latitude } 4151.00 \\ \text { Type } & \text { Type } & \text { Status } & \text { Status } & \text { Longitude } 8929.00\end{array}$

ROR

Factor

Wild/Scenic Protection

Wild/Scenic Tributary or

Upstream/Downstream

wild/Scenic Location

Cultural value

Fish Presence Value

Geologic Value

Historic Value

Other Value

Recreation Value

Scenic Value
IL

Name
PESF*KW Rating (MWh)

12400.00

6200

W
Exist Prob

0.90

Wildlife Value

Threatened/Endangered Fish

Threatened/Endangered Wildlife

0.90 Federal Land Code 103

0.90 Federal Land Code 104

0.90 Federal Land Code 105

0.90 Federal Land Code 106

0.90 Federal Land Code 107

$Y \quad 0.75$ Federal Land Code 108

$Y \quad 0.75$ Federal Land Code 198 Energy Rating (MWh)

0.90
Exist Prob

0.90

0.90

0.90

0.90

0.90

0.90

0.90

0.90

0.90

0.90 
RES OURCE DA TABASE I I S T I N G

DATE : $12 / 06 / 96$

PAGE NO: 37

FERC

Number

Plant Name

Stream

State

ILO11 GRAND DETOUR

ROCK R

Name

II

County Name

LEE

River Basin

ROCK RIVER BASIN

Class Owner Name

F CORPS OF ENGINEERS

\begin{tabular}{|c|c|c|c|c|}
\hline $\begin{array}{l}\text { Name Plate } \\
\text { Rating (KW) }\end{array}$ & PESF & PESF* KW & $\begin{array}{l}\text { Annual Energy } \\
\text { Rating (MWh) }\end{array}$ & $\begin{array}{l}\text { PESF Annual } \\
\text { Energy Rating (MWh) }\end{array}$ \\
\hline 9300.00 & 0.25 & 2325.00 & 52000.00 & 13000 \\
\hline
\end{tabular}

$\begin{array}{lllll}\text { Unit } & \text { Plant } & \text { Project } & \text { Dam } & \text { Latitude } 4154.00 \\ \text { Type } & \text { Type } & \text { Status } & \text { Status } & \text { Longitude } 8927.00\end{array}$

ROR

$\mathrm{U}$

Factor

Wild/Scenic Protection

Wild/Scenic Tributary or Upstream/Downstream wild/Scenic Location

Cultural value

Fish Presence Value

Geologic Value

Historic Value

Other Value

Recreation Value

Scenic Value
Exist Prob

$\longrightarrow$

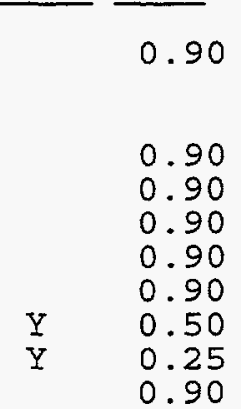

Factor

Wildiife Value Threatened/Endangered Fish Threatened/Endangered Wildlife Federal Land Code 103

Federal Land Code 104

Federal Land Code 105

Federal Land Code 106

Federal Land Code 107

Federal Land Code 108

Federal Land Code 198

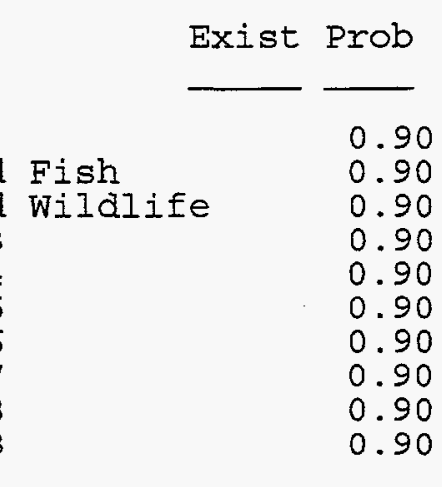


FERC

Number

Plant Name

ILO12 BYRON

County Name

OGLE

\section{Class}

Owner Name

F

CORPS OF ENGINEERS

Name Plate Rating ( $\mathrm{KW}$ )

$$
6800.00
$$
PESF 0.25 1.700 .00
Annual Energy

Type

Plant
Type

ROR

\section{Factor}

Wild/Scenic Protection Wild/Scenic Tributary or Upstream/Downstream wild/Scenic Location Cultural Value

Fish Presence Value

Geologic Value

Historic value

Other Value

Recreation Value

Scenic Value
Project Status

Exist Prob

Factor

Latitude

U

$$
37000.00
$$
Status Longitude 8917.00

State

Name

IL
River Basin

ROCK RIVER BASIN
PESF Annua] Energy Rating (MWh)

9250

4210.00 
RES O U R C D A T A B A S E I I S T I N G

DATE : $12 / 06 / 96$

PAGE NO: 39

FERC

Number

Plant Name

Stream

State

IL013 ROCKTON

ROCK R

Name

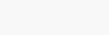

$$
\text { County Name }
$$

River Basin

WINNEBAGO

ROCK RIVER BASIN

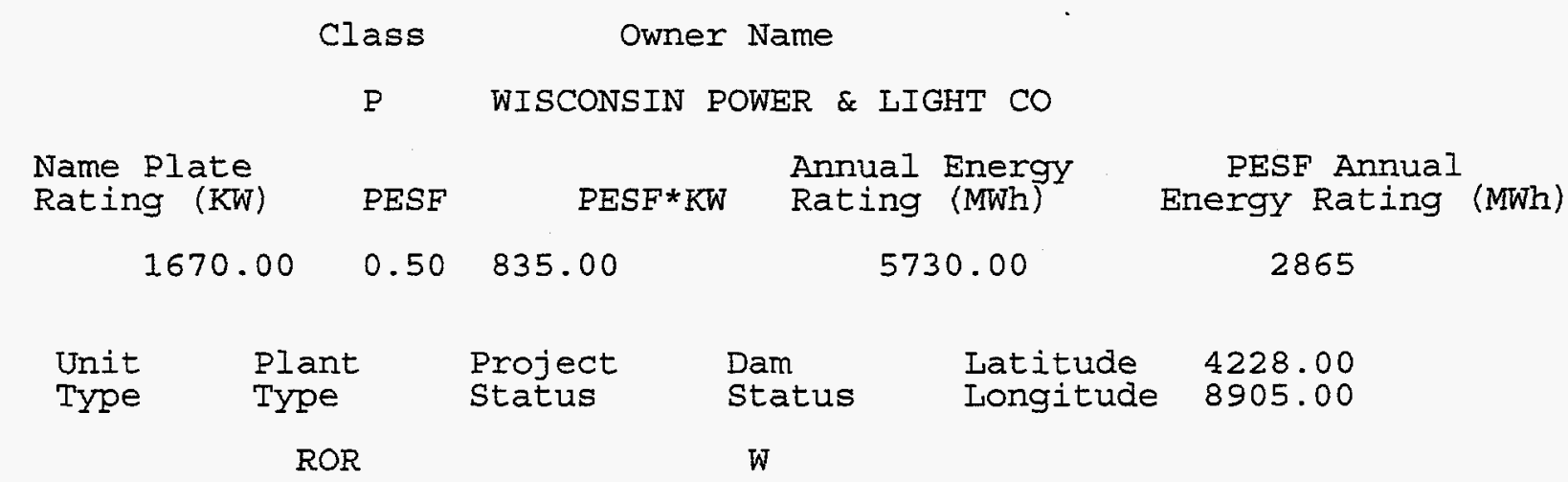

Factor

Wild/Scenic Protection

Wild/Scenic Tributary or Upstream/Downstream wild/Scenic Location Cultural Value Fish Presence Value Geologic Value Historic Value other Value Recreation value Scenic Value
Exist Prob

Factor

0.90 Wildlife Value Threatened/Endangered Fish Threatened/Endangered Wildlife $Y$

0.90 Federal Land Code 103

0.90 Federal Land Code 104

0.90 Federal Land Code 105

0.90 Federal Land Code 1.06

0.90 Federal Land Code 107

Y 0.75 Federal Land Code 108

0.90

0.90
Exist Prob

0.90

0.90

0.75

0.90

0.90

0.90

0.90

0.90

0.90

0.90 

R E S O U R C E
$D A T A$ B A S E
L I S T I N G

DATE : $12 / 06 / 96$

PAGE NO: 40

FERC

Number

Plant Name

ILO14 MOIINE I\&D 15

County Name

ROCK ISILAND

\author{
Stream \\ SYLVAN SLOUGH, MISS R \\ River Basin \\ MISSISSIPPI MAIN STEM
}

State

Name

IL

$\begin{array}{cr}\text { Class } & \text { Owner Name } \\ \mathrm{P} & \text { IOWA ILI G \& E CO }\end{array}$

Name Plate

$\begin{array}{ll} & \text { Annual Energy } \\ \text { PESF } & \text { PESF*KW } \\ \text { Rating (MWh) }\end{array}$

PESF Annual

Rating (KW)

$0.50 \quad 9840.00$

154820.00

Energy Rating (MWh)

19680.00

Project

$\begin{array}{lll}\text { Unit } & \text { Plant } & \text { Projec } \\ \text { Type } & \text { Type } & \text { Status }\end{array}$

$\begin{array}{lll}\text { Dam } & \text { Latitude } & 4131.00 \\ \text { Status } & \text { Longitude } & 9032.00\end{array}$

ROR

W

Factor

Wild/Scenic Protection

wild/Scenic Tributary or Upstream/Downstream wild/Scenic Location

Cultural Value

Fish Presence Value

Geologic Value

Historic Value

Other Value

Recreation value

Scenic Value
Exist Prob

Factor

0.90 Wildlife Value

Threatened/Endangered Fish Threatened/Endangered Wildilife Y

0.90

0.90

0.90

0.90

0.90

$Y \quad 0.75$

$Y \quad 0.75$

Federal Land Code 103

Federal Land Code 104

Federal Land Code 105

Federal Land Code 106

Federal Land Code 107

Federal Land Code 108

Federal Land Code 198

Exist Prob

0.90

0.75

0.75

0.90

0.90

0.90

0.90

0.90

0.90

0.90 
$R E S O U R C E$ D A T A B A E I I S T I N G

DATE : $12 / 06 / 96$

PAGE NO: 41

FERC

Number

Plant Name

Stream

State

Tumber

MISSISSIPPI L\&D 27

MISSISSIPPI $\mathrm{R}$

Name

ILO15

County Name

River Basin

MADISON

MERAMEC RIVER BASIN

Class Owner Name

F CORPS OF ENGINEERS

$\begin{array}{lcccc}\begin{array}{l}\text { Name Plate } \\ \text { Rating (KW) }\end{array} & \text { PESF } & \text { PESF*KW } & \begin{array}{l}\text { Annual Energy } \\ \text { Rating }\end{array} & \begin{array}{c}\text { PESF Annual } \\ \text { Enh) }\end{array} \\ 15340.00 & 0.75 & 11505.00 & 89940.00 & 67455\end{array}$

$\begin{array}{lllll}\text { Unit } & \text { Plant } & \text { Project } & \text { Dam } & \text { Latitude } \\ \text { Type } & \text { Type } & \text { Status } & \text { Status } & \text { Longitude } 9012.00\end{array}$

ROR WO

Factor

Wild/Scenic Protection

Wild/Scenic Tributary or Upstream/Downstream wild/Scenic Location Cultural Value

Fish Presence Value

Geologic Value

Historic Value

Other Value

Recreation Value

Scenic Value

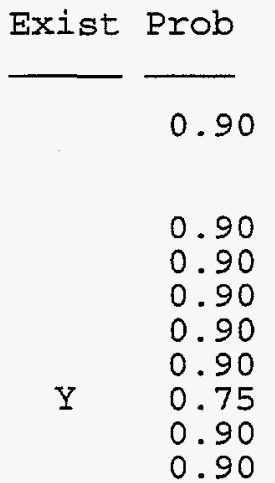

Factor

Exist Prob

Wildlife Value Threatened/Endangered Fish Threatened/Endangered Wildife Federal Iand Code 103 Federal Land Code 104 Federal Land Code 105 Federal Land Code 106 Federal Land Code 107 Federal Land Code 108

Federal Irand Code 198 

R E S O U R C E
D A T A B A S E
I I S T N G

DATE : $12 / 06 / 96$

PAGE NO: 42

FERC

Number

Plant Name

Stream

State

II101 ELGIN, KIMBALL STREET

FOX RIVER

Name

County Name

River Basin

KANE

ILIINOIS RIVER BASIN

Class Owner Name

Name Plate

Rating (KW)

600.00
PESF

$0.75 \quad 450.00$

Unit

Type

\begin{abstract}
Plant
Type
\end{abstract}

ROR

Factor

Wild/Scenic Protection

Wild/Scenic Tributary or

Upstream/Downstream

wild/scenic Location

Cultural Value

Fish Presence Value

Geologic Value

Historic Value

other Value

Recreation Value

Scenic Value

Status

$\mathrm{Y}$
Annual Energy
Rating (MWh)

PESF*KW

2630.00

Project

Dam
Status

Latitude

Longitude

4203.00

WO

Exist Prob

Factor

Exist Prob

0.90

Wildlife Value

Threatened/Endangered Fish

Threatened/Endangered Wildlife

0.90

Federal Land Code 103

0.90 Federal Land Code 104

0.90 Federal Land Code 105

0.90 Federal Land Code 106

0.90 Federal Land Code 107

0.75 Federal Land Code 108

Federal Land Code 198
PESF Annual

Energy Rating (MWh)

1972.50
0.90

$\begin{array}{ll} & \text { Exist Prob } \\ & \\ \text { Fish } & 0.90 \\ \text { Wildife } & 0.90 \\ & 0.90 \\ & 0.90 \\ & 0.90 \\ & 0.90 \\ & 0.90 \\ & 0.90 \\ & 0.90 \\ & 0.90\end{array}$

II

0.90 

$R E S O U R C E$
D A T A B A S E
L I S T I N G

DATE : $12 / 06 / 96$

PAGE NO: 43

FERC

Number

Plant Name

Stream

State

Name

IL102 LAGRANGE

IIIINOIS RIVER

IL

County Name

River Basin

BROWN

ILLINOIS RIVER BASIN

Class Owner Name

\begin{tabular}{cccccc}
$\begin{array}{l}\text { Name Plate } \\
\text { Rating (KW) }\end{array}$ & PESF & \multicolumn{1}{c}{ PESF*KW } & $\begin{array}{l}\text { Annual } \\
\text { Rating }\end{array}$ & $\begin{array}{c}\text { Energy } \\
\text { (MWh) }\end{array}$ & $\begin{array}{c}\text { PESF Annual } \\
\text { Energy Rating (MWh) }\end{array}$ \\
12160.00 & 0.75 & 9120.00 & & 53260.00 & 39945 \\
Unit & Plant & Project & Dam & Latitude & 3957.00 \\
Type & Type & Status & Status & Longitude & 9032.00 \\
& ROR & & wo & &
\end{tabular}

Factor

Exist Prob

Factor

Exist Prob

Wild/Scenic Protection

Wild/Scenic Tributary or Upstream/Downstream wild/Scenic Location

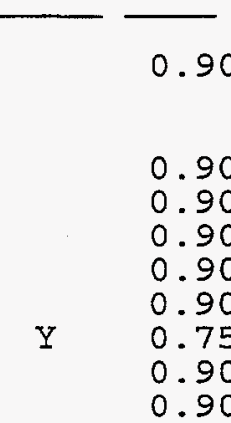

Wildife value

Threatened/Endangered Fish Threatened/Endangered Wildife

0.90 Federal Land Code 103

0.90 Federal Land Code 104

0.90 Federal Land Code 105

0.90 Federal Land Code 106

Fish Presence Value

Geologic Value

Historic Value

other Value

Recreation value

Scenic Value
0.90

Federal Land Code 106

Federal Land Code 108

Federal Land Code 198

Lexist $\begin{aligned} & \text { Prob } \\ & \end{aligned}$


DATE : $12 / 06 / 96$

PAGE NO: 44

FERC

Number

Plant Name

Stream

State

Name

II103 YORKVILLE

FOX RIVER

IL

County Name

River Basin

KENDAIL

ILLINOIS RIVER BASIN

Class Owner Name

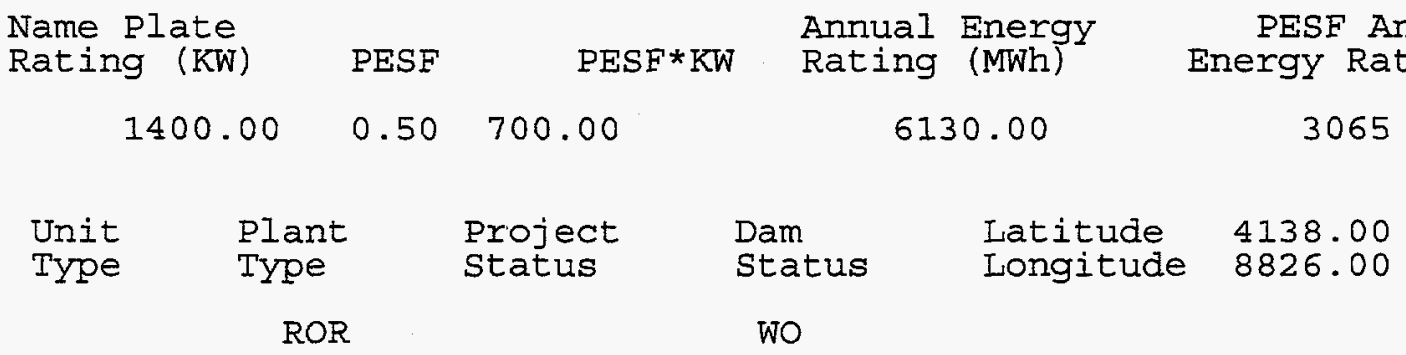

Factor

Exist Prob

Factor

Exist Prob

Wild/Scenic Protection

Wild/Scenic Tributary or

0.90 Wildlife Value

$\begin{array}{lll}\text { Wildilfe Value } & & 0.90 \\ \text { Threatened/Endangered Fish } & Y & 0.75\end{array}$

Upstream/Downstream Threatened/Endangered Wildlife

wild/Scenic Location

0.90 Federal Land Code 103

Cultural value

Fish Presence Value

0.90 Federal Iand Code 104

0.90

0.90

0.90 Federal Land Code 105

0.90

0.90 Federal Iand Code 106

0.90 Federal Land Code 107

0.90

0.90

0.90

other Value

Recreation Value

0.75 Federal Iand Code 108

0.90

Scenic Value

Federal Land Code 198

0.90 
DATE : $12 / 06 / 96$

PAGE NO: 45

FERC

Number

Plant Name

Stream

State

IL104 LAKE DECATUR

SANGAMON RIVER

Name

County Name

River Basin

MACON

ILLINOIS RIVER BASIN

II

Class Owner Name

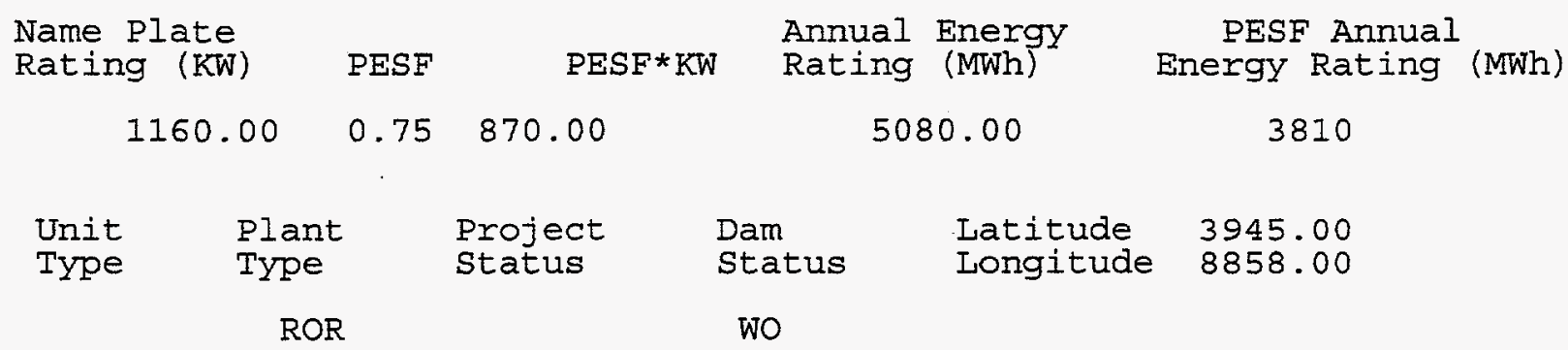

\section{Factor}

Wild/Scenic Protection Wild/Scenic Tributary or Upstream/Downstream wild/Scenic Location Cultural Value

Fish Presence Value

Geologic Value

Historic Value

other Value

Recreation Value

Scenic Value
Exist Prob

0.90

Wildlife Value

Threatened/Endangered Fish

Threatened/Endangered Wildiffe

0.90 Federal Land Code 103

0.90 Federal Land Code 104

0.90 Federal Land Code 105

0.90 Federal Land Code 106

0.90 Federal Land Code 107

$Y \quad 0.75$

0.90

0.90
Federal Land Code 108

Federal Land Code 198
Exist Prob

0.90

0.90

0.90

0.90

0.90

0.90

0.90

0.90

0.90

0.90 
RESOURCE D A TABASE L I S T I N G

DATE : $12 / 06 / 96$

PAGE NO: 46

FERC

Number

Plant Name

Stream

State

IL106 LAKE LOU YAEGER

WEST FORK SHOAL CREEK

Name

County Name

River Basin

MONTGOMERY

KASKASKIA RIVER BASIN

Class Owner Name

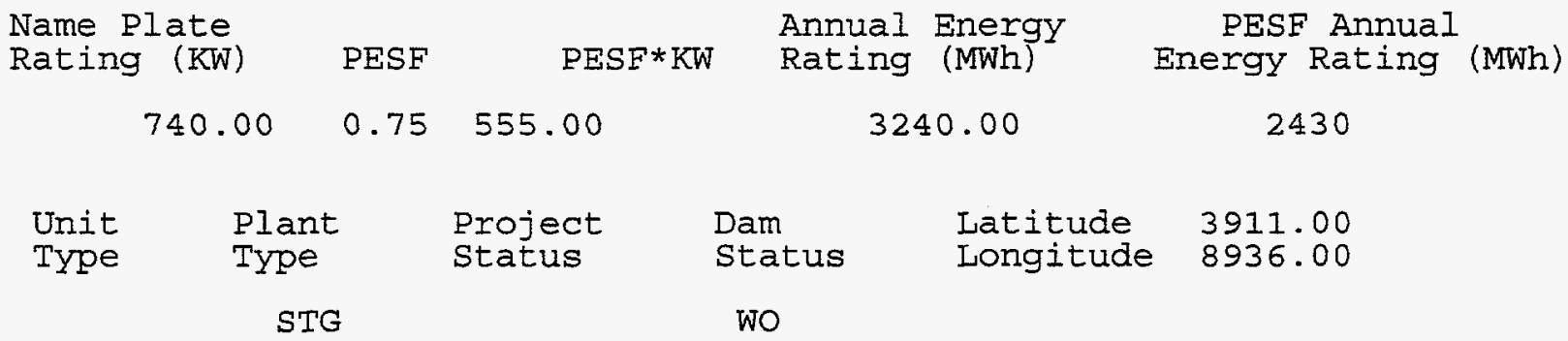

Factor

Wild/Scenic Protection

Wild/scenic Tributary or

Upstream/Downstream

wild/Scenic Location

Cultural Value

Fish Presence Value

Geologic Value

Historic Value

other Value

Recreation Value

Scenic Value
Exist Prob

Factor

Exist Prob

0.90 Wildlife Value

Threatened/Endangered Fish

Threatened/Endangered Wildife

0.90 Federal Land Code 103

0.90 Federal Land Code 104

0.90 Federal Land Code 105

0.90 Federal Land Code 106

0.90 Federal Land Code 107

Y 0.75 Federal Land Code 108

0.90 Federal Land Code $198 \quad 0.90$
0.90

0.90

0.90

0.90

0.90

0.90

0.90

0.90

0.90

0.90
IL 
DATE : $12 / 06 / 96$

PAGE NO: 47

FERC

Number

IL 107
Plant Name

PEORIA

County Name

PEORIA

Class state

Stream

IILINOIS RIVER

IL
River Basin

ILLINOIS RIVER BASIN

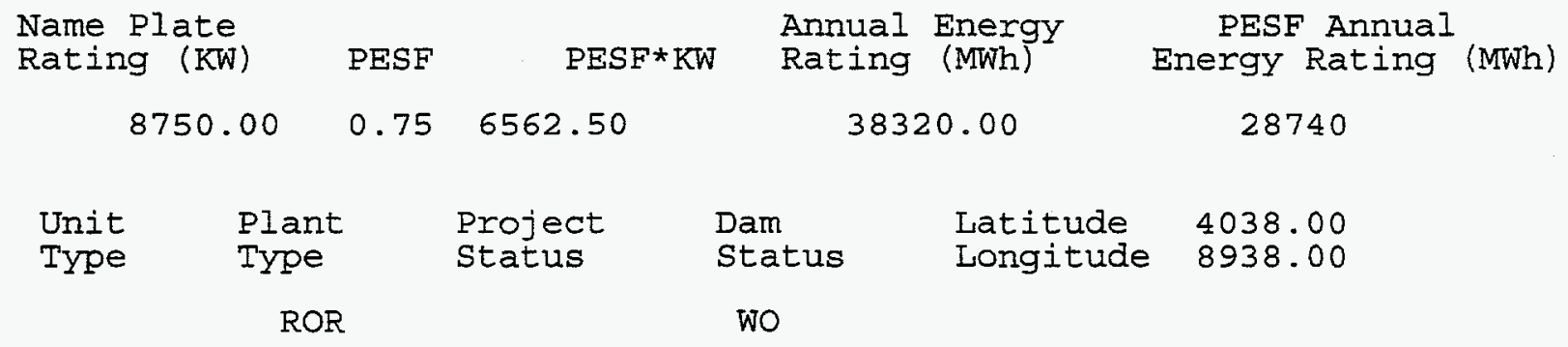

Owner Name

\section{Factor}

Wild/Scenic Protection

Wild/Scenic Tributary or Upstream/Downstream wild/Scenic Location Cultural Value

Fish Presence Value

Geologic Value

Historic Value

other Value

Recreation Value

Scenic Value
Exist Prob

0.90

0.90

0.90

0.90

0.90

0.90

Y

0.75

0.90

0.90
Factor

Exist Prob

Wildlife Value

Threatened/Endangered Fish

Threatened/Endangered Wildlife

Federal Land Code 103

Federal Land Code 104

Federal Iand Code 105

Federal Iand Code 106

Federal Land Code 107

Federal Land Code 108

Federal Land Code 198
0.90

0.90

0.90

0.90

0.90

0.90

0.90

0.90

0.90

0.90 

R E S O U R C E
D A T A B A S E
L I S T I N G

DATE : $12 / 06 / 96$

PAGE NO: 48

FERC

Number

Plant Name

Stream

State

III08 SEARS

ROCK RIVER

Name

County Name

River Basin

ROCK ISLAND

ROCK RIVER BASIN

Class Owner Name

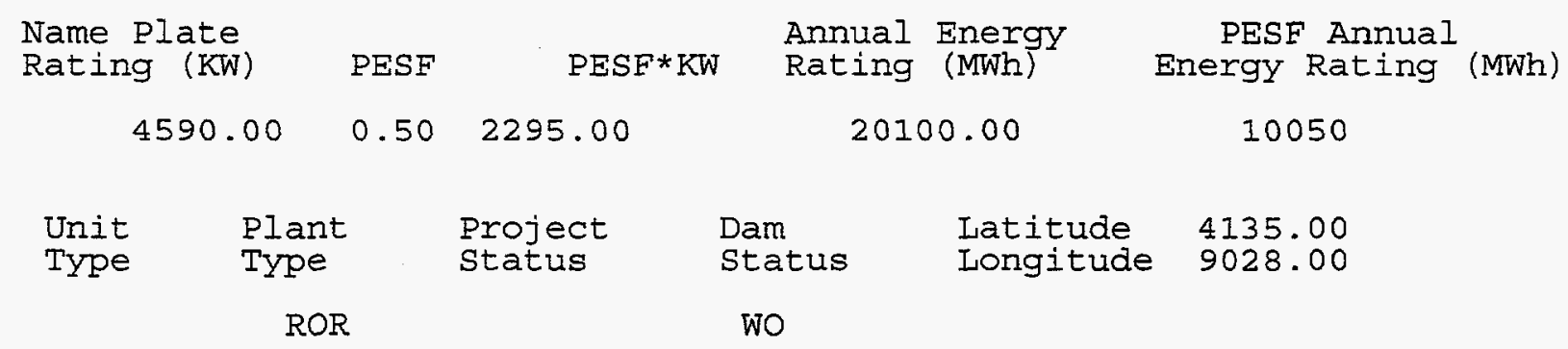

Factor

Exist Prob

Factor

Exist Prob

Wild/Scenic Protection

wild/Scenic Tributary or

Upstream/Downstream

wild/Scenic Location

Cultural Value

Fish Presence Value

Geologic Value

Historic Value

other Value

Recreation Value

Scenic Value

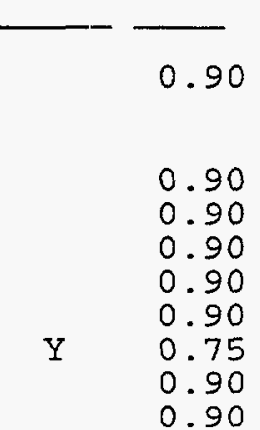

Wildlife Value

Threatened/Endangered Fish Threatened/Endangered Wildlife Y

Federal Land Code 103

Federal Land Code 104

Federal Land Code 105

Federal Land Code 106

Federal Land Code 107

Federal Land Code 108

Federal Land Code 198
IL 
DATE : $12 / 06 / 96$

PAGE NO: 49

FERC

Number

Plant Name

ILI09 LAKE SPRINGFIELD

County Name

SANGAMON

Class

Owner Name
State

Name

SUGAR CREEK

IL

River Basin

ILIINOIS RIVER BASIN

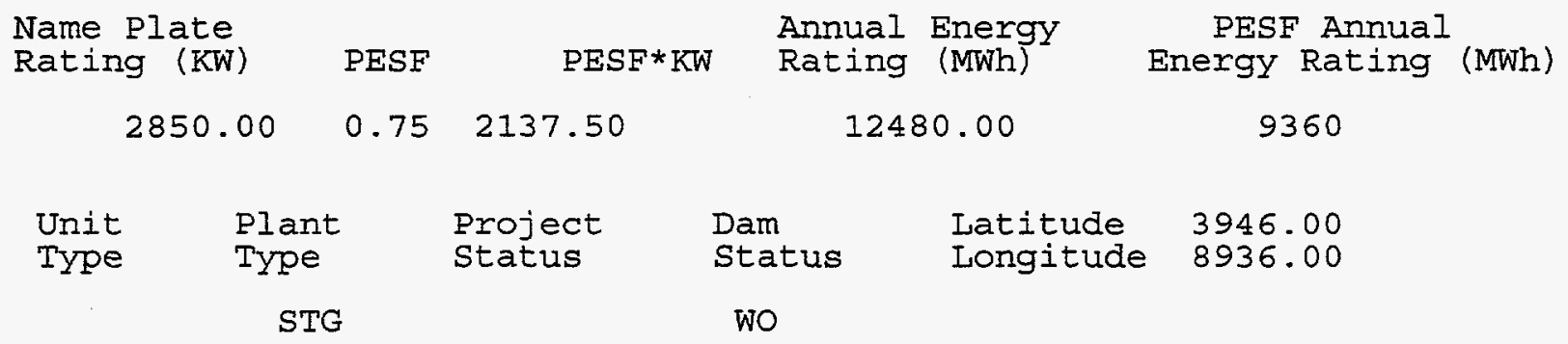

Factor

Exist Prob

Wild/Scenic Protection

wild/Scenic Tributary or Upstream/Downstream wild/Scenic Location Cultural Value

Fish Presence Value

Geologic Value

Historic Value

other Value

Recreation Value

Scenic Value

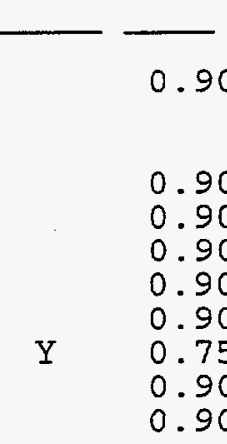

Factor

Exist Prob

$\begin{array}{lrl}\text { Factor } & \text { Exist } & \text { Prob } \\ & & \\ \text { Wildlife Value } & 0.90 \\ \text { Threatened/Endangered Fish } & 0.90 \\ \text { Threatened/Endangered Wildlife } & 0.90 \\ \text { Federal Land Code 103 } & 0.90 \\ \text { Federal Land Code 104 } & 0.90 \\ \text { Federal Land Code 105 } & 0.90 \\ \text { Federal Land Code 106 } & 0.90 \\ \text { Federal Land Code 107 } & 0.90 \\ \text { Federal Land Code 108 } & 0.90 \\ \text { Federal Land Code 198 } & 0.90\end{array}$

